\title{
PERISTALTIC TRANSPORT OF AN OLDROYD-B FLUID IN A PLANAR CHANNEL
}

\author{
T. HAYAT, Y. WANG, K. HUTTER, S. ASGHAR, AND A. M. SIDDIQUI \\ Received 5 June 2003 and in revised form 20 May 2004
}

The effects of an Oldroyd-B fluid on the peristaltic mechanism are examined under the long wavelength assumption. Analytical expressions for the stream function, the axial velocity, and the pressure rise per wavelength are obtained up to the second order in the dimensionless wave number. The effects of the various parameters of interest on the flow are shown and discussed.

\section{Introduction}

The word peristalsis derives from the Greek word $\pi \varepsilon \rho \iota \sigma \tau \alpha \lambda \tau \iota \kappa o s$ which means clasping and compressing. It is used to describe a progressive wave of contraction along a channel or tube whose cross-sectional area consequently varies. Peristalsis is regarded as having considerable relevance in biomechanics and especially as a major mechanism for fluid transport in many biological systems (as it is in the human). It appears in the ureter, in the intestines, and in the oviducts, to name just a few instances.

Great strives have been undertaken, both experimentally and theoretically, to study the propagation of waves in peristaltic motion $[3,12,14,18,26,52,53]$. Arbitrary shapes of these waves [27, 29, 31, 32, 33] as well as sinusoidal waves [1, 10, 12, 15, 23, 49] have been analyzed and measuring techniques $[9,24]$ were designed to test and verify early hydrodynamic models [15, 40, 41].

The governing equations are nonlinear, so assumptions are made about the amplitude ratio, the wavenumber, and the Reynolds number. The amplitude ratio is the ratio of the amplitude of the wave to the half-width of the channel and is usually taken to be small. The case of vanishingly small Reynolds number has also received considerable attention $[3,12,41]$. To include nonlinear effects due to nonvanishing Reynolds number, solutions are usually presented as expansions in terms of a small parameter. They are generally of two types:

(1) expansion parameter is the amplitude of the wave that disturbs the wall; such an expansion was pursued in [15] for the channel and in $[13,55]$ for the pipe, up to second order. Only zero-mean flow was considered for the second-order terms, 
a restriction that was removed in [35]. This approach, while valid for all Reynolds numbers and wavelengths of the disturbing wall, is restricted to small amplitudes and has been applied only to a sinusoidal wave;

(2) expansion in terms of the wavenumber and the Reynolds number for all wave amplitudes. This has been done only for sinusoidal waves, and up to first order in the square of the wavenumber (the natural parameter) and second order in the Reynolds number [22, 28, 56]. The nonsinusoidal wave was studied in [27] for small intestine and for zero Reynolds number only.

To obtain information about flows at moderate Reynolds number it has been necessary to use numerical methods. Several investigators [2, 10, 21, 49] used numerical methods for the solution of the Newtonian hydrodynamical equations. The results, in general, agree with the analytical perturbation solutions in their range of validity with the exception of the calculations of the pressure field by Takabatake and Ayukawa [49]. It is noted that the higher-order terms of Reynolds and wavenumbers do not significantly extend the range of validity of the results.

The application of the theory of particle-fluid mixture is also very useful in understanding a number of diverse physical problems concerning peristalsis. An interesting example is the particulate suspension theory of blood $[5,20,34,36,46,47,50]$. Peristaltic transport of solid particles with fluid has first been attempted in [21]. Various geometric and dynamic effects on the particle transport in a channel with flexible walls were examined. The peristaltic motion for the case of two-phase flow was studied in [48] where a perturbation solution for a small amplitude ratio is given.

Most studies on the peristaltic motion assume the physiological fluids to behave like Newtonian fluids with constant viscosity. However, this approach fails to give an adequate understanding of the peristaltic mechanism involved in small blood vessels, lymphatic vessels, intestine, and ductus efferentes of the male reproductive tracts. In these body organs, the viscosity of the fluid varies across the thickness of the duct $[11,16,19]$. Also, the assumption that the chyme in small intestine is a Newtonian material of variable viscosity is not adequate in reality. Chyme is undoubtedly a non-Newtonian fluid. Some authors (see, e.g., [51]) feel that the main factor responsible for moving the chyme along the intestine is a gradient in the frequency of segmentation (a process of oscillating contraction and relaxation of smooth muscles in the intestine wall) along the length of intestine. Moreover, peristaltic waves die out after travelling a very short distance; peristaltic waves which travel the entire length of small intestine do not occur in humans except under abnormal conditions. Also, in transport of spermatozoa in the cervical canal, there are some other important factors, responsible for the transport of semen in ductus efferentes. One of the major factors is cilia, which keep semen moving towards the epididymis $[7,17,25,30,54]$. The phenomenon of peristalsis has also been proposed as a mechanism for the transport of spermatic fluid (semen) in vas deferens [39]. Movement through vas deferens is accomplished by means of peristaltic action of contractile cells in the duct wall $[39,51]$. However, there is no doubt that peristalsis aids in moving semen in ductus efferentes, the chyme in the intestine, and flow of semen in vas deferens.

The above review of physiological flows indicates that non-Newtonian viscoelastic rheology is the correct way of properly describing the peristaltic flow through channels and 
tubes. Only a few studies $[4,8,42,43,44,45,47]$ have considered this aspect of the problem. Although the second, and third-order models in $[43,44,45]$ take into account normal stress differences and shear-thinning/thickening effects, they lack other features such as stress relaxation. The Oldroyd-B fluid, which includes elastic and memory effects exhibited by dilute solutions, has been extensively used in many applications, and also results of simulations fit experimental data quite well $[6,37,38]$. However, so far, no attempt has been made to understand the peristaltic motion for an Oldroyd-B fluid. We propose to study the effects of an Oldroyd-B fluid on the mechanism of peristaltic transport in a planar channel. Of course the natural coordinate system is axisymmetric; however, the planar case has been predominantly studied. Qualitatively the transport phenomenon of the fluid is similar for both configurations [23]. Also, experimental data are available for channel flows $[26,53]$. Therefore, the present mathematical model considers an Oldroyd-B fluid between parallel walls on which a sinusoidal travelling wave is imposed. The assumption for the present analysis is that the length of the peristaltic wave is large compared with the half-width of the channel. This assumption is similar to those used in $[22,43]$ for the peristaltic motion of Newtonian and second-order fluids, respectively. A regular perturbation technique is adopted to solve the present problem and solutions are expanded in a power series of the small dimensionless wavenumber. The Reynolds number and material time constants are left arbitrary. The analysis is completely analytical but lengthy, and closed-form solutions up to second order of the wavenumber are presented. The effects of the nonlinear terms of the governing equations on the fluid transport are constructed. Comparison is made between the results for the Newtonian and Oldroyd-B fluids. The explicit non-Newtonian terms are obtained and their effect on peristaltic motion is examined. The results for Maxwell and Newtonian fluids are obtained as special cases of the presented analysis.

\section{Basic equations}

Consider an incompressible fluid whose balance laws of mass and linear momentum are given by

$$
\begin{gathered}
\operatorname{div} \overline{\mathbf{V}}=0, \\
\rho \dot{\mathbf{V}}=\operatorname{div} \overline{\mathbf{T}}+\rho \overline{\mathbf{f}},
\end{gathered}
$$

where $\rho, \overline{\mathbf{V}}, \overline{\mathbf{T}}$, and $\overline{\mathbf{f}}$ are mass density, velocity, Cauchy stress tensor, and specific body force and the $\operatorname{dot}(\cdot)$ denotes material time derivative. In the ensuing analysis, body forces will be ignored and isothermal conditions will be implied. The above system of equations will be closed by a constitutive equation for the stress tensor. The constitutive equation for the Cauchy stress $\overline{\mathbf{T}}$ in an Oldroyd-B fluid is given by [37]

$$
\overline{\mathbf{T}}=-\bar{p} \mathbf{I}+\overline{\mathbf{S}},
$$

where the extra stress tensor $\overline{\mathbf{S}}$ is given by

$$
\overline{\mathbf{S}}+\Lambda_{1}\left(\frac{d \overline{\mathbf{S}}}{d \bar{t}}-\overline{\mathbf{L}} \overline{\mathbf{S}}-\overline{\mathbf{S}} \overline{\mathbf{L}}^{T}\right)=\mu\left\{\overline{\mathbf{A}}_{1}+\Lambda_{2}\left(\frac{d \overline{\mathbf{A}}_{1}}{d \bar{t}}-\overline{\mathbf{L}} \overline{\mathbf{A}}_{1}-\overline{\mathbf{A}}_{1} \overline{\mathbf{L}}^{T}\right)\right\}
$$


in which $-\bar{p} \mathbf{I}$ is the spherical part of the stress due to the constraint of incompressibility, $d / d \bar{t}$ denotes material time derivative, $\mu$ is the viscosity, and $\Lambda_{1}$ and $\Lambda_{2}$ are material time constants referred to as relaxation and retardation time, respectively. It is assumed that $\Lambda_{1} \geq \Lambda_{2} \geq 0$. The tensors $\overline{\mathbf{L}}$ and $\overline{\mathbf{A}}_{1}$ are defined as follows:

$$
\overline{\mathbf{L}}=\operatorname{grad} \overline{\mathbf{V}}, \quad \overline{\mathbf{A}}_{1}=\overline{\mathbf{L}}+\overline{\mathbf{L}}^{T},
$$

where $\overline{\mathbf{V}}$ is the velocity vector. It should be noted that this model includes the classical linear case for $\Lambda_{1}=\Lambda_{2}=0$, and when $\Lambda_{2}=0$, the model reduces to the Maxwell model.

\section{Formulation of the problem and flow equations}

Consider a two-dimensional flow of an Oldroyd-B fluid in an infinite channel having width $2 a$. Assume an infinite wave train travelling with velocity $c$ along the walls. Choose a rectangular coordinate system for the channel with $\bar{X}$ along the central line in the direction of wave propagation, and $\bar{Y}$ transverse to it. Let the geometry of the wall surface be defined as

$$
\bar{h}(\bar{X}, \bar{t})=a+b \sin \left[\frac{2 \pi}{\lambda}(\bar{X}-c \bar{t})\right]
$$

where $b$ is the wave amplitude and $\lambda$ the wavelength. Assume, moreover, that there is no motion of the wall in the longitudinal direction (this assumption constrains the deformation of the wall; it does not necessarily imply that the channel is rigid against longitudinal motions, but is a convenient simplification that can be justified by a more complete analysis. The assumption implies that for the no-slip condition $\bar{U}=0$ at the wall).

For unsteady two-dimensional flows,

$$
\overline{\mathbf{V}}=[\bar{U}(\bar{X}, \bar{Y}, \bar{t}), \bar{V}(\bar{X}, \bar{Y}, \bar{t}), 0]
$$

and we find that (2.1)-(2.5), in the absence of body forces, take the following form:

$$
\begin{gathered}
\frac{\partial \bar{U}}{\partial \bar{X}}+\frac{\partial \bar{V}}{\partial \bar{Y}}=0, \\
\rho\left(\frac{\partial}{\partial \bar{t}}+\bar{U} \frac{\partial}{\partial \bar{X}}+\bar{V} \frac{\partial}{\partial \bar{Y}}\right) \bar{U}=-\frac{\partial \bar{p}(\bar{X}, \bar{Y}, \bar{t})}{\partial \bar{X}}+\frac{\partial \bar{S}_{\bar{X} \bar{X}}}{\partial \bar{X}}+\frac{\partial \bar{S}_{\bar{X} \bar{Y}}}{\partial \bar{Y}} \\
\rho\left(\frac{\partial}{\partial \bar{t}}+\bar{U} \frac{\partial}{\partial \bar{X}}+\bar{V} \frac{\partial}{\partial \bar{Y}}\right) \bar{V}=-\frac{\partial \bar{p}(\bar{X}, \bar{Y}, \bar{t})}{\partial \bar{Y}}+\frac{\partial \bar{S}_{\bar{X} \bar{Y}}}{\partial \bar{X}}+\frac{\partial \bar{S}_{\bar{Y} \bar{Y}}}{\partial \bar{Y}}
\end{gathered}
$$




$$
\begin{gathered}
\bar{S}_{\bar{X} \bar{X}}+\Lambda_{1}\left[\left(\frac{\partial}{\partial \bar{t}}+\bar{U} \frac{\partial}{\partial \bar{X}}+\bar{V} \frac{\partial}{\partial \bar{Y}}\right) \bar{S}_{\bar{X} \bar{X}}-2 \frac{\partial \bar{U}_{\bar{X}}}{\partial \bar{X}} \bar{X}_{\bar{X} \bar{X}}-2 \frac{\partial \bar{U}}{\partial \bar{Y}} \bar{S}_{\bar{X} \bar{Y}}\right] \\
=2 \mu \frac{\partial \bar{U}}{\partial \bar{X}}+2 \mu \Lambda_{2}\left[\left(\frac{\partial}{\partial \bar{t}}+\bar{U} \frac{\partial}{\partial \bar{X}}+\bar{V} \frac{\partial}{\partial \bar{Y}}\right) \frac{\partial \bar{U}}{\partial \bar{X}}-2\left(\frac{\partial \bar{U}}{\partial \bar{X}}\right)^{2}-\frac{\partial \bar{U}}{\partial \bar{Y}}\left(\frac{\partial \bar{U}}{\partial \bar{Y}}+\frac{\partial \bar{V}}{\partial \bar{X}}\right)\right] \\
\bar{S}_{\bar{X} \bar{Y}}+\Lambda_{1}\left[\left(\frac{\partial}{\partial \bar{t}}+\bar{U} \frac{\partial}{\partial \bar{X}}+\bar{V} \frac{\partial}{\partial \bar{Y}}\right) \bar{S}_{\bar{X} \bar{Y}}-\frac{\partial \bar{U}}{\partial \bar{Y}} \bar{S}_{\bar{Y} \bar{Y}}-\frac{\partial \bar{V}}{\partial \bar{X}} \bar{S}_{\bar{X} \bar{X}}\right] \\
=\mu\left(\frac{\partial \bar{U}}{\partial \bar{Y}}+\frac{\partial \bar{V}}{\partial \bar{X}}\right)+\mu \Lambda_{2}\left[\left(\frac{\partial}{\partial \bar{t}}+\bar{U} \frac{\partial}{\partial \bar{X}}+\bar{V} \frac{\partial}{\partial \bar{Y}}\right)\left(\frac{\partial \bar{U}}{\partial \bar{Y}}+\frac{\partial \bar{V}}{\partial \bar{X}}\right)\right. \\
\left.-2\left(\frac{\partial \bar{U}}{\partial \bar{X}} \frac{\partial \bar{V}}{\partial \bar{X}}+\frac{\partial \bar{U}}{\partial \bar{Y}} \frac{\partial \bar{V}}{\partial \bar{Y}}\right)\right] \\
\bar{S}_{\bar{Y} \bar{Y}}+\Lambda_{1}\left[\left(\frac{\partial}{\partial \bar{t}}+\bar{U} \frac{\partial}{\partial \bar{X}}+\bar{V} \frac{\partial}{\partial \bar{Y}}\right) \bar{S}_{\bar{Y} \bar{Y}}-2 \frac{\partial \bar{V}}{\partial \bar{X}} \bar{S}_{\bar{X} \bar{Y}}-2 \frac{\partial \bar{V}}{\partial \bar{Y}} \bar{S}_{\bar{Y} \bar{Y}}\right] \\
=2 \mu \frac{\partial \bar{V}}{\partial \bar{Y}}+2 \mu \Lambda_{2}\left[\left(\frac{\partial}{\partial \bar{t}}+\bar{U} \frac{\partial}{\partial \bar{X}}+\bar{V} \frac{\partial}{\partial \bar{Y}}\right) \frac{\partial \bar{V}}{\partial \bar{Y}}-2\left(\frac{\partial \bar{V}}{\partial \bar{Y}}\right)^{2}-\frac{\partial \bar{V}}{\partial \bar{X}}\left(\frac{\partial \bar{U}}{\partial \bar{Y}}+\frac{\partial \bar{V}}{\partial \bar{X}}\right)\right]
\end{gathered}
$$

where $\bar{U}$ and $\bar{V}$ are the longitudinal and transverse velocity components.

In the laboratory frame $(\bar{X}, \bar{Y})$, the flow in the channel is unsteady, but if we choose moving coordinates $(\bar{x}, \bar{y})$ which travel in the positive $\bar{X}$-direction with the same speed as the wave, then the flow can be treated as steady. This coordinate system is known as the wave frame. The coordinate frames are related through

$$
\bar{x}=\bar{X}-c \bar{t}, \quad \bar{y}=\bar{Y},
$$

and the velocity components in the laboratory and wave frames are related by

$$
\bar{u}=\bar{U}-c, \quad \bar{v}=\bar{V},
$$

where $\bar{u}$ and $\bar{v}$ are dimensional velocity components in the directions of $\bar{x}$ and $\bar{y}$, respectively. Employing these transformations in (3), we obtain

$$
\begin{gathered}
\frac{\partial \bar{u}}{\partial \bar{x}}+\frac{\partial \bar{v}}{\partial \bar{y}}=0, \\
\rho\left(\bar{u} \frac{\partial}{\partial \bar{x}}+\bar{v} \frac{\partial}{\partial \bar{y}}\right) \bar{u}=-\frac{\partial \bar{p}}{\partial \bar{x}}+\frac{\partial \bar{S}_{\bar{x} \bar{x}}}{\partial \bar{x}}+\frac{\partial \bar{S}_{\bar{x} \bar{y}}}{\partial \bar{y}}, \\
\rho\left(\bar{u} \frac{\partial}{\partial \bar{x}}+\bar{v} \frac{\partial}{\partial \bar{y}}\right) \bar{v}=-\frac{\partial \bar{p}}{\partial \bar{y}}+\frac{\partial \bar{S}_{\bar{x} \bar{y}}}{\partial \bar{x}}+\frac{\partial \bar{S}_{\bar{y} \bar{y}}}{\partial \bar{y}},
\end{gathered}
$$




$$
\begin{aligned}
& \bar{S}_{\bar{x} \bar{x}}+ \Lambda_{1}\left[\left(\bar{u} \frac{\partial}{\partial \bar{x}}+\bar{v} \frac{\partial}{\partial \bar{y}}\right) \bar{S}_{\bar{x} \bar{x}}-2 \frac{\partial \bar{u}}{\partial \bar{x}} \bar{S}_{\bar{x} \bar{x}}-2 \frac{\partial \bar{u}}{\partial \bar{y}} \bar{S}_{\bar{x} \bar{y}}\right] \\
&= 2 \mu \frac{\partial \bar{U}}{\partial \bar{x}}+2 \mu \Lambda_{2}\left[\left(\bar{u} \frac{\partial}{\partial \bar{x}}+\bar{v} \frac{\partial}{\partial \bar{y}}\right) \frac{\partial \bar{u}}{\partial \bar{x}}-2\left(\frac{\partial \bar{u}}{\partial \bar{x}}\right)^{2}-\frac{\partial \bar{u}}{\partial \bar{y}}\left(\frac{\partial \bar{u}}{\partial \bar{y}}+\frac{\partial \bar{v}}{\partial \bar{x}}\right)\right], \\
& \bar{S}_{\bar{x} \bar{y}}+\Lambda_{1}\left[\left(\bar{u} \frac{\partial}{\partial \bar{x}}+\bar{v} \frac{\partial}{\partial \bar{y}}\right) \bar{S}_{\bar{x} \bar{y}}-\frac{\partial \bar{u}}{\partial \bar{y}} \bar{S}_{\bar{y} \bar{y}}-\frac{\partial \bar{v}}{\partial \bar{x}} \bar{S}_{\bar{x} \bar{x}}\right] \\
&=\mu\left(\frac{\partial \bar{u}}{\partial \bar{y}}+\frac{\partial \bar{v}}{\partial \bar{x}}\right)+\mu \Lambda_{2}\left[\left(\bar{u} \frac{\partial}{\partial \bar{x}}+\bar{v} \frac{\partial}{\partial \bar{y}}\right)\left(\frac{\partial \bar{u}}{\partial \bar{y}}+\frac{\partial \bar{v}}{\partial \bar{x}}\right)-2\left(\frac{\partial \bar{u}}{\partial \bar{x}} \frac{\partial \bar{v}}{\partial \bar{x}}+\frac{\partial \bar{u}}{\partial \bar{y}} \frac{\partial \bar{v}}{\partial \bar{y}}\right)\right], \\
& \bar{S}_{\bar{y} \bar{y}}+\Lambda_{1}\left[\left(\bar{u} \frac{\partial}{\partial \bar{x}}+\bar{v} \frac{\partial}{\partial \bar{y}}\right) \bar{S}_{\bar{y} \bar{y}}-2 \frac{\partial \bar{v}}{\partial \bar{x}} \bar{S}_{\bar{x} \bar{y}}-2 \frac{\partial \bar{v}}{\partial \bar{y}} \bar{S}_{\bar{y} \bar{y}}\right] \\
&=2 \mu \frac{\partial \bar{v}}{\partial \bar{y}}+2 \mu \Lambda_{2}\left[\left(\bar{u} \frac{\partial}{\partial \bar{x}}+\bar{v} \frac{\partial}{\partial \bar{y}}\right) \frac{\partial \bar{v}}{\partial \bar{y}}-2\left(\frac{\partial \bar{v}}{\partial \bar{y}}\right)^{2}-\frac{\partial \bar{v}}{\partial \bar{x}}\left(\frac{\partial \bar{u}}{\partial \bar{y}}+\frac{\partial \bar{v}}{\partial \bar{x}}\right)\right] .
\end{aligned}
$$

The formulation of the boundary conditions is postponed until Section 5.

\section{Dimensionless formulation}

To set the important parameters of the outlined problem in evidence, a scale analysis is performed and the equations are nondimensionalized. Using the dimensionless variables

$$
\begin{gathered}
\bar{x}=\frac{\lambda x}{2 \pi}, \quad \bar{y}=a y, \quad \bar{u}=c u, \quad \bar{v}=c v, \\
\bar{S}=\frac{\mu c}{a} S, \quad \bar{p}=\frac{\lambda \mu c}{2 \pi a^{2}} p, \quad \bar{h}=a h
\end{gathered}
$$

in (3), we arrive at

$$
\begin{gathered}
\delta \frac{\partial u}{\partial x}+\frac{\partial v}{\partial y}=0 \\
\mathscr{R e}\left[\left(\delta u \frac{\partial}{\partial x}+v \frac{\partial}{\partial y}\right) u\right]=-\frac{\partial p}{\partial x}+\delta \frac{\partial S_{x x}}{\partial x}+\frac{\partial S_{x y}}{\partial y} \\
\delta \mathscr{R e}\left[\left(\delta u \frac{\partial}{\partial x}+v \frac{\partial}{\partial y}\right) v\right]=-\frac{\partial p}{\partial y}+\delta^{2} \frac{\partial S_{x y}}{\partial x}+\delta \frac{\partial S_{y y}}{\partial y} \\
S_{x x}+\lambda_{1}\left[\left(\delta u \frac{\partial}{\partial x}+v \frac{\partial}{\partial y}\right) S_{x x}-2 \delta \frac{\partial u}{\partial x} S_{x x}-2 \frac{\partial u}{\partial y} S_{x y}\right] \\
=2 \delta \frac{\partial u}{\partial x}+2 \lambda_{2}\left[\delta\left(\delta u \frac{\partial}{\partial x}+v \frac{\partial}{\partial y}\right) \frac{\partial u}{\partial x}-2 \delta^{2}\left(\frac{\partial u}{\partial x}\right)^{2}-\frac{\partial u}{\partial y}\left(\frac{\partial u}{\partial y}+\delta \frac{\partial v}{\partial x}\right)\right],
\end{gathered}
$$




$$
\begin{gathered}
S_{x y}+\lambda_{1}\left[\left(\delta u \frac{\partial}{\partial x}+v \frac{\partial}{\partial y}\right) S_{x y}-\delta \frac{\partial v}{\partial x} S_{x x}-\frac{\partial u}{\partial y} S_{y y}\right] \\
=\left(\frac{\partial u}{\partial y}+\delta \frac{\partial v}{\partial x}\right)+\lambda_{2}\left[\left(\delta u \frac{\partial}{\partial x}+v \frac{\partial}{\partial y}\right)\left(\frac{\partial u}{\partial y}+\delta \frac{\partial v}{\partial x}\right)-2\left(\delta^{2} \frac{\partial u}{\partial x} \frac{\partial v}{\partial x}+\frac{\partial u}{\partial y} \frac{\partial v}{\partial y}\right)\right], \\
S_{y y}+\lambda_{1}\left[\left(\delta u \frac{\partial}{\partial x}+v \frac{\partial}{\partial y}\right) S_{y y}-2 \delta \frac{\partial v}{\partial x} S_{x y}-2 \frac{\partial v}{\partial y} S_{y y}\right] \\
=2 \frac{\partial v}{\partial y}+2 \lambda_{2}\left[\left(\delta u \frac{\partial}{\partial x}+v \frac{\partial}{\partial y}\right) \frac{\partial v}{\partial y}-2\left(\frac{\partial v}{\partial y}\right)^{2}-\delta \frac{\partial v}{\partial x}\left(\frac{\partial u}{\partial y}+\delta \frac{\partial v}{\partial x}\right)\right],
\end{gathered}
$$

where the dimensionless wavenumber $\delta$, the Reynolds number $\mathscr{R}$ e, and the Weissenberg numbers $\lambda_{1}$ and $\lambda_{2}$ are defined, respectively, as

$$
\delta=\frac{2 \pi a}{\lambda}, \quad \mathscr{R} \mathrm{e}=\frac{c a}{\mu / \rho}, \quad \lambda_{1}=\frac{\Lambda_{1} c}{a}, \quad \lambda_{2}=\frac{\Lambda_{2} c}{a}
$$

These have easy physical interpretations: $\delta$ is a measure of how large the semidepth of the peristaltic motion is, as compared to its wavelength. It is an aspect ratio and thus an expression of shallowness. The Reynolds number $\mathscr{R} e$ is formed with the wave speed, the amplitude, and the kinematic viscosity of the Newtonian part of the constitutive behavior; $\lambda_{1}$ and $\lambda_{2}$ measure the elastic contributions of the stress behavior.

The continuity equation (4.2), after defining the dimensionless stream function $\Psi(x$, y) by the relations

$$
u=\frac{\partial \Psi}{\partial y}, \quad v=-\delta \frac{\partial \Psi}{\partial x}
$$

is identically satisfied and, from (4.3)-(4.7) we deduce

$$
\begin{gathered}
\delta \mathscr{R}\left[\left(\frac{\partial \Psi}{\partial y} \frac{\partial}{\partial x}-\frac{\partial \Psi}{\partial x} \frac{\partial}{\partial y}\right) \frac{\partial \Psi}{\partial y}\right]=-\frac{\partial p}{\partial x}+\delta \frac{\partial S_{x x}}{\partial x}+\frac{\partial S_{x y}}{\partial y} \\
-\delta^{3} \mathscr{R e}\left[\left(\frac{\partial \Psi}{\partial y} \frac{\partial}{\partial x}-\frac{\partial \Psi}{\partial x} \frac{\partial}{\partial y}\right) \frac{\partial \Psi}{\partial x}\right]=-\frac{\partial p}{\partial y}+\delta^{2} \frac{\partial S_{x y}}{\partial x}+\delta \frac{\partial S_{y y}}{\partial y} \\
\delta \mathscr{R e}\left[\left(\frac{\partial \Psi}{\partial y} \frac{\partial}{\partial x}-\frac{\partial \Psi}{\partial x} \frac{\partial}{\partial y}\right)\left(\frac{\partial^{2} \Psi}{\partial y^{2}}+\delta^{2} \frac{\partial^{2} \Psi}{\partial x^{2}}\right)\right] \\
=\delta \frac{\partial^{2}\left(S_{x x}-S_{y y}\right)}{\partial x \partial y}+\left(\frac{\partial^{2}}{\partial y^{2}}-\delta^{2} \frac{\partial^{2}}{\partial x^{2}}\right) S_{x y}
\end{gathered}
$$




$$
\begin{aligned}
& S_{x x}+ \lambda_{1}\left[\delta\left(\frac{\partial \Psi}{\partial y} \frac{\partial}{\partial x}-\frac{\partial \Psi}{\partial x} \frac{\partial}{\partial y}\right) S_{x x}-2 \delta \frac{\partial^{2} \Psi}{\partial x \partial y} S_{x x}-2 \frac{\partial^{2} \Psi}{\partial y^{2}} S_{x y}\right] \\
&= 2 \delta \frac{\partial^{2} \Psi}{\partial x \partial y}+2 \lambda_{2}\left[\delta^{2}\left(\frac{\partial \Psi}{\partial y} \frac{\partial}{\partial x}-\frac{\partial \Psi}{\partial x} \frac{\partial}{\partial y}\right) \frac{\partial^{2} \Psi}{\partial x \partial y}-2 \delta^{2}\left(\frac{\partial^{2} \Psi}{\partial x \partial y}\right)^{2}\right. \\
&\left.-\frac{\partial^{2} \Psi}{\partial y^{2}}\left(\frac{\partial^{2} \Psi}{\partial y^{2}}-\delta^{2} \frac{\partial^{2} \Psi}{\partial x^{2}}\right)\right] \\
& S_{x y}+ \lambda_{1}\left[\delta\left(\frac{\partial \Psi}{\partial y} \frac{\partial}{\partial x}-\frac{\partial \Psi}{\partial x} \frac{\partial}{\partial y}\right) S_{x y}+\delta^{2} \frac{\partial^{2} \Psi}{\partial x^{2}} S_{x x}-\frac{\partial^{2} \Psi}{\partial y^{2}} S_{y y}\right] \\
&=\left(\frac{\partial^{2} \Psi}{\partial y^{2}}-\delta^{2} \frac{\partial^{2} \Psi}{\partial x^{2}}\right)+\lambda_{2}\left[\delta\left(\frac{\partial \Psi}{\partial y} \frac{\partial}{\partial x}-\frac{\partial \Psi}{\partial x} \frac{\partial}{\partial y}\right)\left(\frac{\partial^{2} \Psi}{\partial y^{2}}-\delta^{2} \frac{\partial^{2} \Psi}{\partial x^{2}}\right)\right. \\
&+\left.2 \delta \frac{\partial^{2} \Psi}{\partial x \partial y}\left(\frac{\partial^{2} \Psi}{\partial y^{2}}+\delta^{2} \frac{\partial^{2} \Psi}{\partial x^{2}}\right)\right] \\
& S_{y y}+\lambda_{1}\left[\delta\left(\frac{\partial \Psi}{\partial y} \frac{\partial}{\partial x}-\frac{\partial \Psi}{\partial x} \frac{\partial}{\partial y}\right) S_{y y}+2 \delta \frac{\partial^{2} \Psi}{\partial x \partial y} S_{y y}+2 \delta^{2} \frac{\partial^{2} \Psi}{\partial x^{2}} S_{x y}\right] \\
&\left.+\delta^{2} \frac{\partial^{2} \Psi}{\partial x^{2}}\left(\frac{\partial^{2} \Psi}{\partial y^{2}}-\delta^{2} \frac{\partial^{2} \Psi}{\partial x^{2}}\right)\right] \\
&=-2 \delta \frac{\partial^{2} \Psi}{\partial x \partial y}+2 \lambda_{2}\left[\delta^{2}\left(\frac{\partial \Psi}{\partial y} \frac{\partial}{\partial x}-\frac{\partial \Psi}{\partial x} \frac{\partial}{\partial y}\right)\left(-\frac{\partial^{2} \Psi}{\partial x \partial y}\right)-2 \delta^{2}\left(\frac{\partial^{2} \Psi}{\partial x \partial y}\right)^{2}\right.
\end{aligned}
$$

where the compatibility equation (4.12) is obtained by eliminating $p$ between (4.10) and (4.11); it represents the vorticity transport equation. Notice that (4.10) and (4.11) are formally decoupled from (4.12)-(4.15). So, the latter are used to determine $\Psi$ and $S_{x x}$, $S_{x y}, S_{y y}$, while the former is then employed to determine the pressure field.

\section{Rate of volume flow and boundary conditions}

The dimensional rate of fluid flow in the laboratory frame is given by

$$
Q=\int_{0}^{\bar{h}} \bar{U}(\bar{X}, \bar{Y}, \bar{t}) d \bar{Y},
$$

where $\bar{h}$, the position of the channel wall, is a function of $\bar{X}$ and $\bar{t}$. The rate of fluid flow in the wave frame is given by

$$
q=\int_{0}^{\bar{h}} \bar{u}(\bar{x}, \bar{y}) d \bar{y},
$$

where $\bar{h}$ is now a function of $\bar{x}$ alone. With the help of (3.4) and (3.5), one can show that these two rates are related through

$$
Q=q+c \bar{h}
$$


The time-averaged flow over a period $T$ at a fixed position $\bar{X}$ is given by

$$
\bar{Q}=\frac{1}{T} \int_{0}^{T} Q d t
$$

On using (5.3) in (5.4), we find that

$$
\bar{Q}=q+a c .
$$

If we define the dimensionless mean flows $\Theta$, in the laboratory frame, and $F$, in the wave frame, according to

$$
\Theta=\frac{\bar{Q}}{a c}, \quad F=\frac{q}{a c},
$$

one finds that (5.5) reduces to

$$
\Theta=F+1,
$$

where, according to the first equation of (4.9),

$$
F=\int_{0}^{h} \frac{\partial \Psi}{\partial y} d y=\Psi(h)-\Psi(0) .
$$

If we choose the zero value of the streamline along the central line $(y=0)$

$$
\Psi(0)=0,
$$

then the shape of the wave at the wall boundary is the streamline with value

$$
\Psi(h)=F .
$$

The boundary conditions for the dimensionless stream function in the wave frame are therefore

$$
\begin{aligned}
& \Psi=0 \quad \text { (by convention) } \\
& \frac{\partial^{2} \Psi}{\partial y^{2}}=0 \quad \text { (by symmetry) } \quad \text { on the central line } y=0, \\
& \frac{\partial \Psi}{\partial y}=-1 \quad \text { (no-slip condition) } \quad \text { at the wall } y=h . \\
& \Psi=F
\end{aligned}
$$

We also note that $h$ represents the dimensionless form of the surface of the peristaltic wall which will be chosen as a sinusoidal function, namely,

$$
h(x)=1+\Phi \sin x
$$

where

$$
\Phi=\frac{b}{a}
$$

is the amplitude ratio or the occlusion and $0<\Phi<1$. 


\section{Perturbation solution}

We note that (4.10)-(4.15) are higher-order nonlinear partial differential equations. Therefore, it seems to be impossible to find the solution in closed form for arbitrary values of all parameters. Even for Newtonian fluids [22, 41], all solutions obtained so far are based on the assumption that one or several parameters are zero or small. Following Jaffrin [22], we expand the flow quantities in a power series of the small parameter $\delta$ as follows:

$$
\begin{aligned}
& \Psi=\Psi_{0}+\delta \Psi_{1}+\delta^{2} \Psi_{2}+\cdots, \\
& p=p_{0}+\delta p_{1}+\delta^{2} p_{2}+\cdots, \\
& S=S_{0}+\delta S_{1}+\delta^{2} S_{2}+\cdots, \\
& F=F_{0}+\delta F_{1}+\delta^{2} F_{2}+\cdots,
\end{aligned}
$$

On substituting (6.1) into (4.10)-(4.15) and then collecting terms of equal powers of $\delta$, one obtains the following sets of perturbed equations.

(i) Zeroth-order equations

$$
\begin{gathered}
\frac{\partial^{2} S_{0 x y}}{\partial y^{2}}=0, \\
-\frac{\partial p_{0}}{\partial x}+\frac{\partial S_{0 x y}}{\partial y}=0, \\
-\frac{\partial p_{0}}{\partial y}=0, \\
S_{0 x x}-2 \lambda_{1} \frac{\partial^{2} \Psi_{0}}{\partial y^{2}} S_{0 x y}=-2 \lambda_{2}\left(\frac{\partial^{2} \Psi_{0}}{\partial y^{2}}\right)^{2}, \\
S_{0 x y}-\lambda_{1} \frac{\partial^{2} \Psi_{0}}{\partial y^{2}} S_{0 y y}=\frac{\partial^{2} \Psi_{0}}{\partial y^{2}}, \\
S_{0 y y}=0 .
\end{gathered}
$$

(ii) First-order equations

$$
\begin{gathered}
\mathscr{R e}\left[\left(\frac{\partial \Psi_{0}}{\partial y} \frac{\partial}{\partial x}-\frac{\partial \Psi_{0}}{\partial x} \frac{\partial}{\partial y}\right) \frac{\partial^{2} \Psi_{0}}{\partial y^{2}}\right]=\frac{\partial^{2}\left(S_{0 x x}-S_{0 y y}\right)}{\partial x \partial y}+\frac{\partial^{2}}{\partial y^{2}} S_{1 x y} \\
\mathscr{R e}\left[\left(\frac{\partial \Psi_{0}}{\partial y} \frac{\partial}{\partial x}-\frac{\partial \Psi_{0}}{\partial x} \frac{\partial}{\partial y}\right) \frac{\partial \Psi_{0}}{\partial y}\right]=-\frac{\partial p_{1}}{\partial x}+\frac{\partial S_{0 x x}}{\partial x}+\frac{\partial S_{1 x y}}{\partial y} \\
-\frac{\partial p_{1}}{\partial y}+\frac{\partial S_{0 y y}}{\partial y}=0
\end{gathered}
$$




$$
\begin{gathered}
S_{1 x x}+\lambda_{1}\left[\left(\frac{\partial \Psi_{0}}{\partial y} \frac{\partial}{\partial x}-\frac{\partial \Psi_{0}}{\partial x} \frac{\partial}{\partial y}\right) S_{0 x x}-2 \frac{\partial^{2} \Psi_{0}}{\partial x \partial y} S_{0 x x}-2 \frac{\partial^{2} \Psi_{0}}{\partial y^{2}} S_{1 x y}-2 \frac{\partial^{2} \Psi_{1}}{\partial y^{2}} S_{0 x y}\right] \\
=2 \frac{\partial^{2} \Psi_{0}}{\partial x \partial y}-4 \lambda_{2} \frac{\partial^{2} \Psi_{0}}{\partial y^{2}} \frac{\partial^{2} \Psi_{1}}{\partial y^{2}} \\
S_{1 x y}+\lambda_{1}\left[\left(\frac{\partial \Psi_{0}}{\partial y} \frac{\partial}{\partial x}-\frac{\partial \Psi_{0}}{\partial x} \frac{\partial}{\partial y}\right) S_{0 x y}-\frac{\partial^{2} \Psi_{0}}{\partial y^{2}} S_{1 y y}-\frac{\partial^{2} \Psi_{1}}{\partial y^{2}} S_{0 y y}\right] \\
=\frac{\partial^{2} \Psi_{1}}{\partial y^{2}}+\lambda_{2}\left[\left(\frac{\partial \Psi_{0}}{\partial y} \frac{\partial}{\partial x}-\frac{\partial \Psi_{0}}{\partial x} \frac{\partial}{\partial y}\right) \frac{\partial^{2} \Psi_{0}}{\partial y^{2}}+2 \frac{\partial^{2} \Psi_{0}}{\partial x \partial y} \frac{\partial^{2} \Psi_{0}}{\partial y^{2}}\right] \\
S_{1 y y}+\lambda_{1}\left[\left(\frac{\partial \Psi_{0}}{\partial y} \frac{\partial}{\partial x}-\frac{\partial \Psi_{0}}{\partial x} \frac{\partial}{\partial y}\right) S_{0 y y}+2 \frac{\partial^{2} \Psi_{0}}{\partial x \partial y} S_{0 y y}\right]=-2 \frac{\partial^{2} \Psi_{0}}{\partial x \partial y} .
\end{gathered}
$$

(iii) Second-order equations

$$
\begin{gathered}
\mathscr{R e}\left[\left(\frac{\partial \Psi_{0}}{\partial y} \frac{\partial}{\partial x}-\frac{\partial \Psi_{0}}{\partial x} \frac{\partial}{\partial y}\right) \frac{\partial^{2} \Psi_{1}}{\partial y^{2}}+\left(\frac{\partial \Psi_{1}}{\partial y} \frac{\partial}{\partial x}-\frac{\partial \Psi_{1}}{\partial x} \frac{\partial}{\partial y}\right) \frac{\partial^{2} \Psi_{0}}{\partial y^{2}}\right] \\
=\frac{\partial^{2}\left(S_{1 x x}-S_{1 y y}\right)}{\partial x \partial y}+\frac{\partial^{2} S_{2 x y}}{\partial y^{2}}-\frac{\partial^{2} S_{0 x y}}{\partial x^{2}}, \\
\mathscr{R e}\left[\left(\frac{\partial \Psi_{0}}{\partial y} \frac{\partial}{\partial x}-\frac{\partial \Psi_{0}}{\partial x} \frac{\partial}{\partial y}\right) \frac{\partial \Psi_{1}}{\partial y}+\left(\frac{\partial \Psi_{1}}{\partial y} \frac{\partial}{\partial x}-\frac{\partial \Psi_{1}}{\partial x} \frac{\partial}{\partial y}\right) \frac{\partial \Psi_{0}}{\partial y}\right] \\
=-\frac{\partial p_{2}}{\partial x}+\frac{\partial S_{1 x x}}{\partial x}+\frac{\partial S_{2 x y}}{\partial y}, \\
S_{2 x x}+\lambda_{1}\left[\left(\frac{\partial \Psi_{0}}{\partial y} \frac{\partial}{\partial x}-\frac{\partial \Psi_{0}}{\partial x} \frac{\partial}{\partial y}\right) S_{1 x x}+\left(\frac{\partial \Psi_{0 x y}}{\partial y}+\frac{\partial S_{1 y y}}{\partial y}=0,\right.\right. \\
\left.-2 \frac{\partial^{2} \Psi_{0}}{\partial x \partial y} S_{1 x x}-2 \frac{\partial^{2} \Psi_{1}}{\partial x \partial y} S_{0 x x}-2 \frac{\partial^{2} \Psi_{0}}{\partial y^{2}} S_{2 x y}-2 \frac{\partial}{\partial y}\right) S_{0 x x} \\
\left.+\frac{\partial^{2} \Psi_{1}}{\partial \Psi_{1}} S_{1 x y}-2 \frac{\partial^{2} \Psi_{2}}{\partial y^{2}} S_{0 x y}\right] \\
+2 \lambda_{2}\left[\left(\frac{\partial \Psi_{0}}{\partial y} \frac{\partial}{\partial x}-\frac{\partial \Psi_{0}}{\partial x} \frac{\partial}{\partial y}\right) \frac{\partial^{2} \Psi_{0}}{\partial x \partial y}-2\left(\frac{\partial^{2} \Psi_{0}}{\partial x \partial y}\right)^{2}-2 \frac{\partial^{2} \Psi_{0}}{\partial y^{2}} \frac{\partial^{2} \Psi_{2}}{\partial y^{2}}\right. \\
\left.=\left(\frac{\partial^{2} \Psi_{1}}{\partial y^{2}}\right)^{2}\right],
\end{gathered}
$$




$$
\begin{aligned}
S_{2 x y}+\lambda_{1}[ & \left(\frac{\partial \Psi_{1}}{\partial y} \frac{\partial}{\partial x}-\frac{\partial \Psi_{1}}{\partial x} \frac{\partial}{\partial y}\right) S_{0 x y}+\left(\frac{\partial \Psi_{0}}{\partial y} \frac{\partial}{\partial x}-\frac{\partial \Psi_{0}}{\partial x} \frac{\partial}{\partial y}\right) S_{1 x y} \\
& \left.-\frac{\partial^{2} \Psi_{0}}{\partial y^{2}} S_{2 y y}-\frac{\partial^{2} \Psi_{1}}{\partial y^{2}} S_{1 y y}-\frac{\partial^{2} \Psi_{2}}{\partial y^{2}} S_{0 y y}+\frac{\partial^{2} \Psi_{0}}{\partial x^{2}} S_{0 x x}\right] \\
= & \frac{\partial^{2} \Psi_{2}}{\partial y^{2}}-\frac{\partial^{2} \Psi_{0}}{\partial x^{2}}+\lambda_{2}\left[\left(\frac{\partial \Psi_{0}}{\partial y} \frac{\partial}{\partial x}-\frac{\partial \Psi_{0}}{\partial x} \frac{\partial}{\partial y}\right) \frac{\partial^{2} \Psi_{1}}{\partial y^{2}}+\left(\frac{\partial \Psi_{1}}{\partial y} \frac{\partial}{\partial x}-\frac{\partial \Psi_{1}}{\partial x} \frac{\partial}{\partial y}\right) \frac{\partial^{2} \Psi_{0}}{\partial y^{2}}\right. \\
& \left.+2 \frac{\partial^{2} \Psi_{0}}{\partial x \partial y} \frac{\partial^{2} \Psi_{1}}{\partial y^{2}}+2 \frac{\partial^{2} \Psi_{1}}{\partial x \partial y} \frac{\partial^{2} \Psi_{0}}{\partial y^{2}}\right] \\
S_{2 y y}+\lambda_{1}\left[\left(\frac{\partial \Psi_{1}}{\partial y} \frac{\partial}{\partial x}-\frac{\partial \Psi_{1}}{\partial x} \frac{\partial}{\partial y}\right) S_{0 y y}+\left(\frac{\partial \Psi_{0}}{\partial y} \frac{\partial}{\partial x}-\frac{\partial \Psi_{0}}{\partial x} \frac{\partial}{\partial y}\right) S_{1 y y}\right. & \left.+2 \frac{\partial^{2} \Psi_{0}}{\partial x^{2}} S_{0 x y}+2 \frac{\partial^{2} \Psi_{0}}{\partial x \partial y} S_{1 y y}+2 \frac{\partial^{2} \Psi_{1}}{\partial x \partial y} S_{0 y y}\right] \\
= & -2 \frac{\partial^{2} \Psi_{1}}{\partial x \partial y}-2 \lambda_{2}\left[\left(\frac{\partial \Psi_{0}}{\partial y} \frac{\partial}{\partial x}-\frac{\partial \Psi_{0}}{\partial x} \frac{\partial}{\partial y}\right) \frac{\partial^{2} \Psi_{0}}{\partial x \partial y}+2\left(\frac{\partial^{2} \Psi_{0}}{\partial x \partial y}\right)^{2}-\frac{\partial^{2} \Psi_{0}}{\partial x^{2}} \frac{\partial^{2} \Psi_{0}}{\partial y^{2}}\right]
\end{aligned}
$$

Because the boundary conditions (5.11), (5.12) are linear, identical conditions fall on every order system of equations; this is why they are not repeated above.

After lengthy calculations (the interested reader may consult the principal author) with (6.2)-(6.14) and the boundary conditions (5.11), (5.12) at each order, the following boundary value problems for the stream function, pressure and stress components are deduced.

(i) Zeroth-order system

$$
\begin{gathered}
\frac{\partial^{4} \Psi_{0}}{\partial y^{4}}=0 \\
\frac{\partial p_{0}}{\partial x}=\frac{\partial^{3} \Psi_{0}}{\partial y^{3}} \\
\frac{\partial p_{0}}{\partial y}=0 \\
S_{0 x x}=2\left(\lambda_{1}-\lambda_{2}\right)\left(\frac{\partial^{2} \Psi_{0}}{\partial y^{2}}\right)^{2} \\
S_{0 x y}=\frac{\partial^{2} \Psi_{0}}{\partial y^{2}} \\
S_{0 y y}=0
\end{gathered}
$$

with boundary conditions

$$
\begin{gathered}
\Psi_{0}=0, \quad \frac{\partial^{2} \Psi_{0}}{\partial y^{2}}=0 \quad \text { at } y=0, \\
\Psi_{0}=F_{0}, \quad \frac{\partial \Psi_{0}}{\partial y}=-1 \quad \text { at } y=h .
\end{gathered}
$$


(ii) First-order system

$$
\begin{gathered}
\mathscr{R e}\left[\left(\frac{\partial \Psi_{0}}{\partial y} \frac{\partial}{\partial x}-\frac{\partial \Psi_{0}}{\partial x} \frac{\partial}{\partial y}\right) \frac{\partial^{2} \Psi_{0}}{\partial y^{2}}\right]-2\left(\lambda_{1}-\lambda_{2}\right) \frac{\partial^{2}}{\partial x \partial y}\left[\left(\frac{\partial^{2} \Psi_{0}}{\partial y^{2}}\right)^{2}\right] \\
=\frac{\partial^{4} \Psi_{1}}{\partial y^{4}}-\left(\lambda_{1}-\lambda_{2}\right) \frac{\partial^{2}}{\partial y^{2}}\left[\left(\frac{\partial \Psi_{0}}{\partial y} \frac{\partial}{\partial x}-\frac{\partial \Psi_{0}}{\partial x} \frac{\partial}{\partial y}\right) \frac{\partial^{2} \Psi_{0}}{\partial y^{2}}\right] \\
-2\left(\lambda_{1}-\lambda_{2}\right) \frac{\partial^{2}}{\partial y^{2}}\left[\frac{\partial^{2} \Psi_{0}}{\partial y^{2}} \frac{\partial^{2} \Psi_{0}}{\partial x \partial y}\right] \\
\frac{\partial p_{1}}{\partial x}=-\mathscr{R e}\left[\left(\frac{\partial \Psi_{0}}{\partial y} \frac{\partial}{\partial x}-\frac{\partial \Psi_{0}}{\partial x} \frac{\partial}{\partial y}\right) \frac{\partial \Psi_{0}}{\partial y}\right]+2\left(\lambda_{1}-\lambda_{2}\right) \frac{\partial}{\partial x}\left[\left(\frac{\partial^{2} \Psi_{0}}{\partial y^{2}}\right)^{2}\right] \\
+\frac{\partial^{3} \Psi_{1}}{\partial y^{3}}-\left(\lambda_{1}-\lambda_{2}\right) \frac{\partial}{\partial y}\left[\left(\frac{\partial \Psi_{0}}{\partial y} \frac{\partial}{\partial x}-\frac{\partial \Psi_{0}}{\partial x} \frac{\partial}{\partial y}\right) \frac{\partial^{2} \Psi_{0}}{\partial y^{2}}\right] \\
-2\left(\lambda_{1}-\lambda_{2}\right) \frac{\partial}{\partial y}\left[\frac{\partial^{2} \Psi_{0}}{\partial y^{2}} \frac{\partial^{2} \Psi_{0}}{\partial x \partial y}\right], \\
S_{1 x y}=\frac{\partial^{2} \Psi_{1}}{\partial y^{2}}-\left(\lambda_{1}-\lambda_{2}\right)\left[\left(\frac{\partial \Psi_{0}}{\partial y} \frac{\partial}{\partial x}-\frac{\partial \Psi_{0}}{\partial x} \frac{\partial}{\partial y}\right) \frac{\partial^{2} \Psi_{0}}{\partial y^{2}}\right]-2\left(\lambda_{1}-\lambda_{2}\right) \frac{\partial^{2} \Psi_{0}}{\partial y^{2}} \frac{\partial^{2} \Psi_{0}}{\partial x \partial y} \\
S_{1 x x}=-2 \lambda_{1}\left(\lambda_{1}-\lambda_{2}\right)\left[\left(\frac{\partial \Psi_{0}}{\partial y} \frac{\partial}{\partial x}-\frac{\partial \Psi_{0}}{\partial x} \frac{\partial}{\partial y}\right)\left(\frac{\partial^{2} \Psi_{0}}{\partial y^{2}}\right)^{2}\right]+4\left(\lambda_{1}-\lambda_{2}\right) \frac{\partial^{2} \Psi_{0}}{\partial y^{2}} \frac{\partial^{2} \Psi_{1}}{\partial y^{2}} \\
\left.\lambda_{1}\right) \frac{\partial^{2} \Psi_{0}}{\partial y^{2}}\left[\left(\frac{\partial \Psi_{0}}{\partial y} \frac{\partial}{\partial x}-\frac{\partial \Psi_{0}}{\partial x} \frac{\partial}{\partial y}\right) \frac{\partial^{2} \Psi_{0}}{\partial y^{2}}\right]+2 \frac{\partial^{2} \Psi_{0}}{\partial x \partial y}, \\
\frac{\lambda^{2} \Psi_{0}}{\partial x \partial y} \\
-\lambda_{1}
\end{gathered}
$$

with boundary conditions

$$
\begin{aligned}
& \Psi_{1}=0, \quad \frac{\partial^{2} \Psi_{1}}{\partial y^{2}}=0 \quad \text { at } y=0, \\
& \Psi_{1}=F_{1}, \quad \frac{\partial \Psi_{1}}{\partial y}=0 \quad \text { at } y=h .
\end{aligned}
$$


(iii) Second-order system

$$
\begin{aligned}
& \mathscr{R e}\left[\left(\frac{\partial \Psi_{0}}{\partial y} \frac{\partial}{\partial x}-\frac{\partial \Psi_{0}}{\partial x} \frac{\partial}{\partial y}\right) \frac{\partial^{2} \Psi_{1}}{\partial y^{2}}+\left(\frac{\partial \Psi_{1}}{\partial y} \frac{\partial}{\partial x}-\frac{\partial \Psi_{1}}{\partial x} \frac{\partial}{\partial y}\right) \frac{\partial^{2} \Psi_{0}}{\partial y^{2}}\right] \\
& +2 \lambda_{1}\left(\lambda_{1}-\lambda_{2}\right) \frac{\partial^{2}}{\partial x \partial y}\left[\left(\frac{\partial \Psi_{0}}{\partial y} \frac{\partial}{\partial x}-\frac{\partial \Psi_{0}}{\partial x} \frac{\partial}{\partial y}\right)\left(\frac{\partial^{2} \Psi_{0}}{\partial y^{2}}\right)^{2}\right]-3 \frac{\partial^{2}}{\partial x \partial y}\left[\frac{\partial^{2} \Psi_{0}}{\partial x \partial y}\right] \\
& +2 \lambda_{1}\left(\lambda_{1}-\lambda_{2}\right) \frac{\partial^{2}}{\partial x \partial y}\left[\frac{\partial^{2} \Psi_{0}}{\partial y^{2}}\left[\left(\frac{\partial \Psi_{0}}{\partial y} \frac{\partial}{\partial x}-\frac{\partial \Psi_{0}}{\partial x} \frac{\partial}{\partial y}\right) \frac{\partial^{2} \Psi_{0}}{\partial y^{2}}\right]\right] \\
& -4\left(\lambda_{1}-\lambda_{2}\right) \frac{\partial^{2}}{\partial x \partial y}\left[\frac{\partial^{2} \Psi_{0}}{\partial y^{2}} \frac{\partial^{2} \Psi_{1}}{\partial y^{2}}\right] \\
& =\frac{\partial^{2} S_{2 x y}}{\partial y^{2}} \\
& \frac{\partial p_{2}}{\partial x}=-\mathscr{R e}\left[\left(\frac{\partial \Psi_{0}}{\partial y} \frac{\partial}{\partial x}-\frac{\partial \Psi_{0}}{\partial x} \frac{\partial}{\partial y}\right) \frac{\partial \Psi_{1}}{\partial y}+\left(\frac{\partial \Psi_{1}}{\partial y} \frac{\partial}{\partial x}-\frac{\partial \Psi_{1}}{\partial x} \frac{\partial}{\partial y}\right) \frac{\partial \Psi_{0}}{\partial y}\right] \\
& -2 \lambda_{1}\left(\lambda_{1}-\lambda_{2}\right) \frac{\partial}{\partial x}\left[\left(\frac{\partial \Psi_{0}}{\partial y} \frac{\partial}{\partial x}-\frac{\partial \Psi_{0}}{\partial x} \frac{\partial}{\partial y}\right)\left(\frac{\partial^{2} \Psi_{0}}{\partial y^{2}}\right)^{2}\right]+2 \frac{\partial}{\partial x}\left[\frac{\partial^{2} \Psi_{0}}{\partial x \partial y}\right] \\
& -2 \lambda_{1}\left(\lambda_{1}-\lambda_{2}\right) \frac{\partial}{\partial x}\left[\frac{\partial^{2} \Psi_{0}}{\partial y^{2}}\left[\left(\frac{\partial \Psi_{0}}{\partial y} \frac{\partial}{\partial x}-\frac{\partial \Psi_{0}}{\partial x} \frac{\partial}{\partial y}\right) \frac{\partial^{2} \Psi_{0}}{\partial y^{2}}\right]\right] \\
& +4\left(\lambda_{1}-\lambda_{2}\right) \frac{\partial}{\partial x}\left[\frac{\partial^{2} \Psi_{0}}{\partial y^{2}} \frac{\partial^{2} \Psi_{1}}{\partial y^{2}}\right]+\frac{\partial S_{2 x y}}{\partial y}
\end{aligned}
$$

$$
\frac{\partial p_{2}}{\partial y}=-\frac{\partial}{\partial y}\left[\frac{\partial^{2} \Psi_{0}}{\partial x \partial y}\right]
$$

$S_{2 x x}+\lambda_{1}\left[\left(\frac{\partial \Psi_{0}}{\partial y} \frac{\partial}{\partial x}-\frac{\partial \Psi_{0}}{\partial x} \frac{\partial}{\partial y}\right) S_{1 x x}+2\left(\lambda_{1}-\lambda_{2}\right)\left(\frac{\partial \Psi_{1}}{\partial y} \frac{\partial}{\partial x}-\frac{\partial \Psi_{1}}{\partial x} \frac{\partial}{\partial y}\right)\left(\frac{\partial^{2} \Psi_{0}}{\partial y^{2}}\right)^{2}\right.$

$$
\begin{array}{r}
-2 \frac{\partial^{2} \Psi_{0}}{\partial x \partial y} S_{1 x x}-4\left(\lambda_{1}-\lambda_{2}\right) \frac{\partial^{2} \Psi_{1}}{\partial x \partial y}\left(\frac{\partial^{2} \Psi_{0}}{\partial y^{2}}\right)^{2}-2 \frac{\partial^{2} \Psi_{0}}{\partial y^{2}} S_{2 x y} \\
\left.-2 \frac{\partial^{2} \Psi_{1}}{\partial y^{2}} S_{1 x y}-2 \frac{\partial^{2} \Psi_{0}}{\partial y^{2}} \frac{\partial^{2} \Psi_{2}}{\partial y^{2}}\right] \\
=2 \frac{\partial^{2} \Psi_{1}}{\partial x \partial y}+2 \lambda_{2}\left[\left(\frac{\partial \Psi_{0}}{\partial y} \frac{\partial}{\partial x}-\frac{\partial \Psi_{0}}{\partial x} \frac{\partial}{\partial y}\right) \frac{\partial^{2} \Psi_{0}}{\partial x \partial y}-2\left(\frac{\partial^{2} \Psi_{0}}{\partial x \partial y}\right)^{2}\right. \\
\left.-2 \frac{\partial^{2} \Psi_{0}}{\partial y^{2}} \frac{\partial^{2} \Psi_{2}}{\partial y^{2}}+\frac{\partial^{2} \Psi_{0}}{\partial x^{2}} \frac{\partial^{2} \Psi_{0}}{\partial y^{2}}-\left(\frac{\partial^{2} \Psi_{1}}{\partial y^{2}}\right)^{2}\right],
\end{array}
$$




$$
\begin{aligned}
S_{2 x y}= & \lambda_{1}\left[\frac{\partial^{2} \Psi_{0}}{\partial y^{2}} S_{2 y y}+\frac{\partial^{2} \Psi_{1}}{\partial y^{2}} S_{1 y y}-2\left(\lambda_{1}-\lambda_{2}\right) \frac{\partial^{2} \Psi_{0}}{\partial x^{2}}\left(\frac{\partial^{2} \Psi_{0}}{\partial y^{2}}\right)^{2}\right. \\
& \left.-\left(\frac{\partial \Psi_{1}}{\partial y} \frac{\partial}{\partial x}-\frac{\partial \Psi_{1}}{\partial x} \frac{\partial}{\partial y}\right) \frac{\partial^{2} \Psi_{0}}{\partial y^{2}}-\left(\frac{\partial \Psi_{0}}{\partial y} \frac{\partial}{\partial x}-\frac{\partial \Psi_{0}}{\partial x} \frac{\partial}{\partial y}\right) S_{1 x y}\right] \\
+ & \frac{\partial^{2} \Psi_{2}}{\partial y^{2}}-\frac{\partial^{2} \Psi_{0}}{\partial x^{2}}+\lambda_{2}\left[\left(\frac{\partial \Psi_{0}}{\partial y} \frac{\partial}{\partial x}-\frac{\partial \Psi_{0}}{\partial x} \frac{\partial}{\partial y}\right) \frac{\partial^{2} \Psi_{1}}{\partial y^{2}}+\left(\frac{\partial \Psi_{1}}{\partial y} \frac{\partial}{\partial x}-\frac{\partial \Psi_{1}}{\partial x} \frac{\partial}{\partial y}\right) \frac{\partial^{2} \Psi_{0}}{\partial y^{2}}\right. \\
& \left.+2 \frac{\partial^{2} \Psi_{0}}{\partial x \partial y} \frac{\partial^{2} \Psi_{1}}{\partial y^{2}}+2 \frac{\partial^{2} \Psi_{1}}{\partial x \partial y} \frac{\partial^{2} \Psi_{0}}{\partial y^{2}}\right], \\
S_{2 y y}= & 2\left(\lambda_{1}-\lambda_{2}\right)\left[\left(\frac{\partial \Psi_{0}}{\partial y} \frac{\partial}{\partial x}-\frac{\partial \Psi_{0}}{\partial x} \frac{\partial}{\partial y}\right) \frac{\partial^{2} \Psi_{0}}{\partial x \partial y}-\frac{\partial^{2} \Psi_{0}}{\partial x^{2}} \frac{\partial^{2} \Psi_{0}}{\partial y^{2}}+2\left(\frac{\partial^{2} \Psi_{0}}{\partial x \partial y}\right)^{2}\right]-2 \frac{\partial^{2} \Psi_{1}}{\partial x \partial y}
\end{aligned}
$$

with boundary conditions

$$
\begin{aligned}
& \Psi_{2}=0, \quad \frac{\partial^{2} \Psi_{2}}{\partial y^{2}}=0 \quad \text { at } y=0, \\
& \Psi_{2}=F_{2}, \quad \frac{\partial \Psi_{2}}{\partial y}=0 \quad \text { at } y=h .
\end{aligned}
$$

We will now solve each system, subject to the boundary conditions, and thereby generate the series solution.

Zeroth-order solution. Mere inspection of the governing equations (6.15)-(6.21) shows that with the exception of (6.18) this zeroth-order problem is described by the properties of a linear viscous fluid flowing such that the long wavelength approximation is satisfied. Only $S_{0 x x}$ depends on the difference of the Weissenberg numbers and therefore exhibits non-Newtonian properties. Effects of this contribution are not visible except possibly on the walls if tractions are measurable. The reader may check that the solution of (6.15)(6.21) in terms of stream function $\Psi_{0}$, the axial velocity $u_{0}$, and the longitudinal pressure gradient $d p_{0} / d x$ is given by

$$
\begin{gathered}
\Psi_{0}=-\frac{3}{2}\left(F_{0}+h\right)\left(\frac{L^{3}}{3}-L\right)-y, \\
u_{0}=-\frac{3}{2 h}\left(F_{0}+h\right)\left(L^{2}-1\right)-1, \\
\frac{d p_{0}}{d x}=-3\left(\frac{F_{0}}{h^{3}}+\frac{1}{h^{2}}\right),
\end{gathered}
$$

where $L=y / h$, and from (6.17) it is clear that the transverse pressure gradient is zero. 
It is also of some interest to calculate the pressure rise over a wavelength $\left(\triangle P_{\lambda}\right)$ in the longitudinal direction on the axis $(y=0)$. At this order, we have

$$
\triangle P_{\lambda_{0}}=\int_{0}^{2 \pi} \frac{d p_{0}}{d x} d x=-3\left[F_{0} I_{3}+I_{2}\right]
$$

where

$$
\begin{gathered}
I_{2}=\frac{2 \pi}{\left(1-\Phi^{2}\right)^{3 / 2}}, \quad I_{3}=\frac{\pi\left(2+\Phi^{2}\right)}{\left(1-\Phi^{2}\right)^{5 / 2}}, \\
I_{n}=\int_{0}^{2 \pi} \frac{d x}{h^{n}}=\frac{1}{1-\Phi^{2}}\left[\left(\frac{2 n-3}{n-1}\right) I_{n-1}-\left(\frac{n-2}{n-1}\right) I_{n-2}\right], \quad n \geq 4 .
\end{gathered}
$$

As already pointed out, expressions (6.36)-(6.39) are the same as for Newtonian fluids [41]. We also remark that the solution for an Oldroyd-B fluid in case of infinite wavelength $(\delta=0)$ is identical to the zeroth-order solution.

First-order solution. Inspection of (6.22)-(6.28) would lead us to assume that nonNewtonian effects will now enter the first-order solution; however, on substituting the zeroth-order solution (6.36) into (6.22) and (6.23), we find that

$$
\begin{gathered}
\frac{\partial^{4} \Psi_{1}}{\partial y^{4}}=\frac{120 b_{1} y^{3}}{h^{7}}+\frac{24 b_{2} y}{h^{5}} \\
\frac{d p_{1}}{d x}=\frac{\partial^{3} \Psi_{1}}{\partial y^{3}}+\mathscr{R e} \frac{d \tilde{p}_{1}}{d x}+2\left(\lambda_{1}-\lambda_{2}\right) \frac{d \hat{p}_{1}}{d x},
\end{gathered}
$$

where

$$
\begin{aligned}
& \frac{d \tilde{p}_{1}}{d x}=-\left[\frac{3 h_{x}\left(3 F_{0}+2 h\right)\left(F_{0}+h\right)}{2 h^{4}}\left\{\frac{y^{4}}{h^{3}}-\frac{3 y^{2}}{2}\left(\frac{y^{3}}{h^{3}}-\frac{1}{h}\right)\right\}\right. \\
& \left.-\frac{9 h_{x} F_{0}\left(F_{0}+h\right)}{2 h^{2}}\left\{\frac{y^{2}}{h^{3}}-\frac{1}{2}\left(\frac{y^{3}}{h^{3}}-\frac{1}{h}\right)\right\}-\frac{3 y^{2} h_{x}\left(3 F_{0}+2 h\right)}{2 h^{4}}+\frac{3 h_{x} F_{0}}{2 h^{2}}\right], \\
& \frac{d \hat{p}_{1}}{d x}=-\left[\frac{32 b_{01}^{2} y^{2} h_{x}}{h^{9}}+\frac{3 b_{01}\left(b_{01 x}-a_{01}\right) y^{2}}{h^{8}}+\frac{\left(b_{02} b_{01 x}-a_{02} b_{01}\right)}{h^{6}}-\frac{12 b_{01}^{2} y^{2} h_{x}}{h^{9}}\right. \\
& \left.-\frac{4 b_{01} b_{02} h_{x}}{h^{7}}-\frac{27 y^{2} h_{x}\left(3 F_{0}+2 h\right)\left(F_{0}+h\right)}{2 h^{7}}+\frac{9 h_{x} F_{0}\left(F_{0}+h\right)}{2 h^{5}}\right], \\
& b_{1}=-\mathscr{R} \frac{h_{x}}{40}\left[3 F_{0}^{2}+5 F_{0} h+2 h^{2}\right], \\
& b_{2}=\mathscr{R e} \frac{h_{x}}{8}\left[3 F_{0}^{2}+3 F_{0} h+h^{2}\right] \text {, } \\
& a_{01}=\frac{h_{x}\left(3 F_{0}+2 h\right)}{2}, \quad a_{02}=-\frac{3 h_{x} F_{0}}{2} \\
& b_{01}=-\frac{3 h\left(F_{0}+h\right)}{2}, \quad b_{02}=\frac{h\left(3 F_{0}+h\right)}{2},
\end{aligned}
$$


and the subscript $x$ denotes differentiation with respect to $x$. Obviously, $\Psi_{1}$ cannot depend on non-Newtonian behavior, but $p_{1}$ may through the last term on the right-hand side of (6.42).

The solution of (6.41) subject to the boundary conditions (6.28) is given by

$$
\Psi_{1}=b_{1}\left(\frac{L^{7}}{7}-L\right)+b_{2}\left(\frac{L^{5}}{5}-L\right)+b_{3}\left(\frac{L^{3}}{3}-L\right)
$$

where $b_{1}$ and $b_{2}$ are defined in (6.45), while $b_{3}$ is given by

$$
b_{3}=-\left[\frac{9}{7} b_{1}+\frac{6}{5} b_{2}+\frac{3}{2} F_{1}\right]=-\mathscr{R} \mathrm{e} \frac{3 h_{x}}{280}\left[33 F_{0}^{2}+27 F_{0} h+8 h^{2}\right]-\frac{3}{2} F_{1} .
$$

The axial velocity and the longitudinal pressure gradient at this order take the form

$$
\begin{gathered}
u_{1}=\frac{1}{h}\left[b_{1}\left(L^{6}-1\right)+b_{2}\left(L^{4}-1\right)+b_{3}\left(L^{2}-1\right)\right], \\
\frac{d p_{1}}{d x}=\frac{30 b_{1} y^{4}}{h^{7}}+\frac{12 b_{2} y^{2}}{h^{5}}+\frac{2 b_{3}}{h^{3}}+\mathscr{R e} \frac{d \tilde{p}_{1}}{d x}+2\left(\lambda_{1}-\lambda_{2}\right) \frac{d \hat{p}_{1}}{d x} .
\end{gathered}
$$

These formulas demonstrate that $u_{1}$ shows no non-Newtonian response but $d p_{1} / d x$ does.

The pressure rise over a wavelength for this order turns out to be

$$
\begin{aligned}
\triangle P_{\lambda_{1}}= & \frac{2 \mathscr{R} \mathrm{e}}{35} \underbrace{\int_{0}^{2 \pi}\left[\frac{27 F_{0}^{2}}{h^{3}}+\frac{3 F_{0}}{h^{2}}-\frac{3}{h}\right] h_{x} d x}_{=0} \\
& -3 F_{1} \int_{0}^{2 \pi} \frac{d x}{h^{3}}-6\left(\lambda_{1}-\lambda_{2}\right) \underbrace{\int_{0}^{2 \pi}\left[\frac{3 F_{0}^{2}}{h^{5}}-\frac{3 F_{0}}{h^{4}}+\frac{1}{2 h^{3}}\right] h_{x} d x}_{=0} \\
= & -3 F_{1} I_{3} .
\end{aligned}
$$

We note that this expression is independent of the non-Newtonian effects; this comes somewhat as a surprise.

Second-order solution. We have solved the second-order system. Computations are very massive and mere inspection of the equations no longer suffices to deduce qualitative 
behavior. We must restrict ourselves to presenting only the key steps and results, respectively. Inserting the zeroth-order and first-order solutions in (6.29), we obtain

$$
\begin{aligned}
& \frac{\partial^{4} \Psi_{2}}{\partial y^{4}}=\mathscr{R} \mathrm{e} \tilde{b}_{1}+2 \lambda_{1}\left(\lambda_{1}-\lambda_{2}\right) \frac{\partial \tilde{b}_{2}}{\partial y}-4\left(\lambda_{1}-\lambda_{2}\right) \frac{\partial \tilde{b}_{3}}{\partial y}+2 \lambda_{1}\left(\lambda_{1}-\lambda_{2}\right) \frac{\partial \tilde{b}_{4}}{\partial y}-\frac{\partial \tilde{b}_{5}}{\partial y} \\
& -2 \lambda_{1}\left(\lambda_{1}-\lambda_{2}\right) \frac{\partial^{2} \tilde{b}_{6}}{\partial y^{2}}+2\left(\lambda_{1}-\lambda_{2}\right) \frac{\partial^{2} \tilde{b}_{7}}{\partial y^{2}}+2\left(\lambda_{1}-\lambda_{2}\right) \frac{\partial^{2} \tilde{b}_{8}}{\partial y^{2}}+2 \lambda_{1}\left(\lambda_{1}-\lambda_{2}\right) \frac{\partial^{2} \tilde{b}_{9}}{\partial y^{2}} \\
& +\left(\lambda_{1}-\lambda_{2}\right) \frac{\partial^{2} \tilde{b}_{1}}{\partial y^{2}}-\lambda_{1}\left(\lambda_{1}-\lambda_{2}\right) \frac{\partial^{2} \tilde{b}_{10}}{\partial y^{2}}-2 \lambda_{1}\left(\lambda_{1}-\lambda_{2}\right) \frac{\partial^{2} \tilde{b}_{11}}{\partial y^{2}}+\tilde{b}_{12} \\
& \frac{\partial p_{2}}{\partial x}=\frac{\partial^{3} \Psi_{2}}{\partial y^{3}}+\mathscr{R} \mathrm{e} \frac{\partial \tilde{p}_{2}}{\partial x}+\mathscr{R} e \frac{\partial \hat{p}_{2}}{\partial x}+\mathscr{R} \frac{\partial p_{2}^{*}}{\partial x}-2 \lambda_{1}\left(\lambda_{1}-\lambda_{2}\right) \frac{\partial}{\partial x}\left(\frac{d_{11} y^{4}}{h^{12}}+\frac{d_{12} y^{2}}{h^{10}}\right) \\
& +4\left(\lambda_{1}-\lambda_{2}\right) \frac{\partial}{\partial x}\left(\frac{e_{11} y^{6}}{h^{6}}+\frac{e_{12} y^{4}}{h^{4}}+\frac{e_{13} y^{2}}{h^{2}}\right)+\frac{\partial}{\partial x}\left[h_{x}\left\{\frac{3\left(3 F_{0}+2 h\right) y^{2}}{h^{4}}-\frac{3 F_{0}}{h^{2}}\right\}\right] \\
& -2 \lambda_{1}\left(\lambda_{1}-\lambda_{2}\right) \frac{\partial}{\partial x}\left[4 b_{01}^{2}\left(b_{01 x}-a_{01}\right) \frac{y^{4}}{h^{12}}+4 b_{01}\left(b_{02} b_{01 x}-a_{02} b_{01}\right) \frac{y^{2}}{h^{10}}\right. \\
& \left.-\frac{16 b_{01}^{3} y^{4} h_{x}}{h^{13}}-\frac{16 b_{01}^{2} b_{02} y^{2} h_{x}}{h^{11}}\right] \\
& +2 \lambda_{1}\left(\lambda_{1}-\lambda_{2}\right) \frac{\partial \tilde{b}_{6}}{\partial y}-2\left(\lambda_{1}-\lambda_{2}\right) \frac{\partial \tilde{b}_{7}}{\partial y}-2\left(\lambda_{1}-\lambda_{2}\right) \frac{\partial \tilde{b}_{8}}{\partial y}-2 \lambda_{1}\left(\lambda_{1}-\lambda_{2}\right) \frac{\partial \tilde{b}_{9}}{\partial y} \\
& -\left(\lambda_{1}-\lambda_{2}\right) \frac{\partial \tilde{b}_{1}}{\partial y}+\lambda_{1}\left(\lambda_{1}-\lambda_{2}\right) \frac{\partial \tilde{b}_{10}}{\partial y}+2 \lambda_{1}\left(\lambda_{1}-\lambda_{2}\right) \frac{\partial \tilde{b}_{11}}{\partial y} \\
& -\frac{\partial}{\partial y}\left\{y^{3}\left[\frac{a_{01 x}}{h^{4}}-\frac{4 a_{01} h_{x}}{h^{5}}\right]+y\left[\frac{a_{02 x}}{h^{2}}-\frac{2 a_{02} h_{x}}{h^{3}}\right]\right\}, \\
& \frac{\partial p_{2}}{\partial y}=-\frac{3 y h_{x}\left(3 F_{0}+2 h\right)}{h^{4}}
\end{aligned}
$$

where

$$
\begin{aligned}
& \tilde{b}_{1}=c_{11} L^{7}+c_{12} L^{5}+c_{13} L^{3}+c_{14} L, \\
& \tilde{b}_{2}=y^{4}\left[\frac{d_{11 x}}{h^{12}}-\frac{12 d_{11} h_{x}}{h^{13}}\right]+y^{2}\left[\frac{d_{12 x}}{h^{10}}-\frac{10 d_{12} h_{x}}{h^{11}}\right], \\
& \tilde{b}_{3}=e_{14} L^{6}+e_{15} L^{4}+e_{16} L^{2}, \\
& \tilde{b}_{4}=y^{4}\left[\frac{d_{13 x}}{h^{12}}-\frac{12 d_{13} h_{x}}{h^{13}}\right]+y^{2}\left[\frac{d_{14 x}}{h^{10}}-\frac{10 d_{14} h_{x}}{h^{11}}\right], \\
& \tilde{b}_{5}=12 y^{2}\left[\frac{a_{01 x}}{h^{4}}-\frac{4 a_{01} h_{x}}{h^{5}}\right]+4\left[\frac{a_{02 x}}{h^{2}}-\frac{2 a_{02} h_{x}}{h^{3}}\right], \\
& \tilde{b}_{6}=\frac{2 b_{01} g_{14} y^{5}}{h^{12}}+\frac{2 b_{01} g_{15} y^{3}}{h^{8}}+\frac{2 b_{01} g_{16} y}{h^{4}},
\end{aligned}
$$




$$
\begin{aligned}
& \tilde{b}_{7}=\frac{14 b_{01} a_{11} y^{7}}{h^{11}}+\frac{10 b_{01} a_{12} y^{5}}{h^{9}}+\frac{6 b_{01} a_{13} y^{3}}{h^{7}}+\frac{2 b_{01} a_{14} y}{h^{5}}, \\
& \tilde{b}_{8}=\frac{18 a_{01} b_{1} y^{7}}{h^{11}}+\frac{6\left(a_{02} b_{1}+2 a_{01} b_{2}\right) y^{5}}{h^{9}}+\frac{2\left(2 a_{02} b_{2}+3 a_{01} b_{3}\right) y^{3}}{h^{7}}+\frac{2 a_{02} b_{3} y}{h^{5}} \text {, } \\
& \tilde{b}_{9}=\frac{4 b_{01}^{2} y^{5}}{h^{8}}\left[\frac{a_{01 x}}{h^{4}}-\frac{4 a_{01} h_{x}}{h^{5}}\right]+\frac{4 b_{01}^{2} y^{3}}{h^{8}}\left[\frac{a_{02 x}}{h^{2}}-\frac{2 a_{02} h_{x}}{h^{3}}\right] \text {, } \\
& \tilde{b}_{10}=\frac{g_{11} y^{5}}{h^{12}}+\frac{g_{12} y^{3}}{h^{10}}+\frac{g_{13} y}{h^{8}}, \quad \tilde{b}_{11}=J_{11} L^{5}+J_{12} L^{3}+J_{13} L, \\
& \tilde{b}_{12}=12 y\left[\frac{a_{01 x}}{h^{4}}-\frac{4 a_{01} h_{x}}{h^{5}}\right] \text {, } \\
& \frac{\partial \tilde{p}_{2}}{\partial x}=\left[\left\{\frac{3\left(F_{0}+h\right)}{2}\left(\frac{y^{2}}{h^{3}}-\frac{1}{h}\right)+1\right\}\right. \\
& \times\left\{b_{1 x}\left(\frac{y^{6}}{h^{7}}-\frac{1}{h}\right)+b_{2 x}\left(\frac{y^{4}}{h^{5}}-\frac{1}{h}\right)+b_{3 x}\left(\frac{y^{2}}{h^{3}}-\frac{1}{h}\right)+b_{1}\left(-7 \frac{y^{6}}{h^{8}}+\frac{1}{h^{2}}\right) h_{x}\right. \\
& \left.\left.+b_{2}\left(-5 \frac{y^{4}}{h^{6}}+\frac{1}{h^{2}}\right) h_{x}+b_{3}\left(-3 \frac{y^{2}}{h^{4}}+\frac{1}{h^{2}}\right) h_{x}\right\}\right] \text {, } \\
& \frac{\partial \hat{p}_{2}}{\partial x}=h_{x}\left[\left\{\frac{\left(3 F_{0}+2 h\right) y^{3}}{2 h^{4}}-\frac{3 y F_{0}}{2 h^{2}}\right\}\left\{\frac{6 b_{1} y^{5}}{h^{7}}+\frac{4 b_{2} y^{3}}{h^{5}}+\frac{2 b_{3} y}{h^{3}}\right\}\right] \\
& -h_{x}\left[\left\{\frac{3\left(3 F_{0}+2 h\right) y^{2}}{2 h^{4}}-\frac{3 F_{0}}{2 h^{2}}\right\}\left\{b_{1}\left(\frac{y^{6}}{h^{7}}-\frac{1}{h}\right)+b_{2}\left(\frac{y^{4}}{h^{5}}-\frac{1}{h}\right)+b_{3}\left(\frac{y^{2}}{h^{3}}-\frac{1}{h}\right)\right\}\right] \text {, } \\
& \frac{\partial p_{2}^{*}}{\partial x}=-\left\{b_{1 x}\left(\frac{y^{7}}{7 h^{7}}-\frac{y}{h}\right)+b_{2 x}\left(\frac{y^{5}}{5 h^{5}}-\frac{y}{h}\right)+b_{3 x}\left(\frac{y^{3}}{3 h^{3}}-\frac{y}{h}\right)\right. \\
& \left.-b_{1}\left(\frac{y^{7}}{h^{8}}-\frac{y}{h^{2}}\right) h_{x}-b_{2}\left(\frac{y^{5}}{h^{6}}-\frac{y}{h^{2}}\right) h_{x}-b_{3}\left(\frac{y^{3}}{h^{4}}-\frac{y}{h^{2}}\right) h_{x}\right\}\left\{\frac{3 y\left(F_{0}+h\right)}{h^{3}}\right\}, \\
& a_{11}=-\frac{h_{x} b_{1}}{h}+\frac{b_{1 x}}{7}=\mathscr{R e}\left(h_{x}\right)^{2}\left[\frac{3 F_{0}^{2}}{40 h}+\frac{3 F_{0}}{28}+\frac{h}{28}\right]-\frac{\mathscr{R e} h_{x x}}{280}\left[3 F_{0}^{2}+5 F_{0} h+2 h^{2}\right] \text {, } \\
& a_{12}=-\frac{h_{x} b_{2}}{h}+\frac{b_{2 x}}{5}=-\mathscr{R e}\left(h_{x}\right)^{2}\left[\frac{3 F_{0}^{2}}{8 h}+\frac{3 F_{0}}{10}+\frac{3 h}{40}\right]+\frac{\mathscr{R e} h_{x x}}{40}\left[3 F_{0}^{2}+3 F_{0} h+h^{2}\right] \text {, } \\
& a_{13}=-\frac{h_{x} b_{3}}{h}+\frac{b_{3 x}}{3}=\frac{\mathscr{R e}\left(h_{x}\right)^{2}}{280}\left[\frac{99 F_{0}^{2}}{h}+54 F_{0}+8 h\right]+\frac{3 F_{1} h_{x}}{2 h} \\
& -\frac{\mathscr{R e} h_{x x}}{280}\left[33 F_{0}^{2}+27 F_{0} h+8 h^{2}\right] \\
& a_{14}=\frac{h_{x}\left(b_{1}+b_{2}+b_{3}\right)}{h}-\left(b_{1 x}+b_{2 x}+b_{3 x}\right) \\
& =-\frac{\mathscr{R e}\left(h_{x}\right)^{2}}{280}\left[\frac{15 F_{0}^{2}}{h}-3 h\right]-\frac{3 F_{1} h_{x}}{2 h}+\frac{\mathscr{R} e h_{x x}}{280}\left[15 F_{0}^{2}+11 F_{0} h+3 h^{2}\right],
\end{aligned}
$$




$$
\begin{aligned}
& c_{11}=\frac{1}{h^{4}}\left[40 a_{11} b_{01}-24 a_{01} b_{1}\right] \text {, } \\
& c_{12}=\frac{1}{h^{4}}\left[42 a_{11} b_{02}+18 b_{01} a_{12}-30 a_{02} b_{1}-6 a_{01} b_{2}\right] \text {, } \\
& c_{13}=\frac{1}{h^{4}}\left[20 a_{12} b_{02}+4 b_{01} a_{13}-12 a_{02} b_{2}+4 a_{01} b_{3}\right] \text {, } \\
& c_{14}=\frac{1}{h^{4}}\left[6 b_{02} a_{13}-2 a_{02} b_{3}-6 a_{01}\left(b_{1}+b_{2}+b_{3}\right)-2 b_{01} a_{14}\right] \text {, } \\
& d_{11}=8\left[b_{01}^{2} b_{01 x}-a_{01} b_{01}^{2}\right]-\frac{4 b_{01}^{3} h_{x}}{h}, \quad d_{12}=8\left[b_{01} b_{02} b_{01 x}-a_{02} b_{01}^{2}\right]-\frac{4 b_{01}^{2} b_{02} h_{x}}{h}, \\
& d_{13}=4\left[b_{01}^{2} b_{01 x}-a_{01} b_{01}^{2}\right]-\frac{16 b_{01}^{3} h_{x}}{h}, \quad d_{14}=4\left[b_{01} b_{02} b_{01 x}-a_{02} b_{01}^{2}\right]-\frac{16 b_{01}^{2} b_{02} h_{x}}{h}, \\
& e_{11}=\frac{12 b_{1} b_{01}}{h^{5}}, \quad e_{12}=\frac{8 b_{2} b_{01}}{h^{5}}, \quad e_{13}=\frac{4 b_{3} b_{01}}{h^{5}}, \\
& e_{14}=e_{11 x}-\frac{6 e_{11} h_{x}}{h}, \quad e_{15}=e_{12 x}-\frac{4 e_{12} h_{x}}{h}, \quad e_{16}=e_{13 x}-\frac{2 e_{13} h_{x}}{h}, \\
& f_{11}=2 b_{01}\left[b_{01 x}-a_{01}-\frac{4 b_{01} h_{x}}{h}\right], \quad f_{12}=2\left[b_{02} b_{01 x}-a_{02} b_{01}-\frac{4 b_{02} b_{01} h_{x}}{h}\right] \text {, } \\
& g_{11}=b_{01} f_{11 x}-\frac{8 b_{01} f_{11} h_{x}}{h}-3 a_{01} f_{11} \text {, } \\
& g_{12}=b_{02} f_{11 x}-\frac{8 b_{02} f_{11} h_{x}}{h}+b_{01} f_{12 x}-\frac{6 b_{01} f_{12} h_{x}}{h}-3 a_{02} f_{11}-a_{01} f_{12}, \\
& g_{13}=b_{02} f_{12 x}-\frac{6 b_{02} f_{12} h_{x}}{h}-a_{02} f_{12}, \quad g_{14}=b_{01}\left[a_{01 x}-\frac{4 a_{01} h_{x}}{h}\right]+18 a_{01}^{2}, \\
& g_{15}=3 b_{02}\left[\frac{a_{01 x}}{h^{2}}-\frac{4 a_{01} h_{x}}{h^{3}}\right]-b_{01}\left[\frac{a_{02 x}}{h^{2}}-\frac{2 a_{02} h_{x}}{h^{3}}\right]+\frac{12 a_{01} a_{02}}{h^{2}} \text {, } \\
& g_{16}=\frac{b_{02}}{h^{2}}\left[\frac{a_{02 x}}{h^{2}}-\frac{2 a_{02} h_{x}}{h^{3}}\right]+\frac{2 a_{02}^{2}}{h^{4}} \text {, } \\
& J_{11}=\frac{6}{h^{7}}\left[a_{01 x} b_{01}^{2}+a_{01} b_{01} b_{01 x}-3 b_{01} a_{01}^{2}-\frac{8 a_{01} b_{01}^{2} h_{x}}{h}\right] \text {, } \\
& J_{12}=\frac{2}{h^{7}}\left[a_{02 x} b_{01}^{2}+a_{02} b_{01} b_{01 x}-\frac{6 a_{02} b_{01}^{2} h_{x}}{h}+3 a_{01 x} b_{01} b_{02}+3 a_{01} b_{01 x} b_{02}\right. \\
& \left.-\frac{24 a_{01} b_{01} b_{02} h_{x}}{h}-10 a_{01} b_{01} a_{02}\right] \\
& J_{13}=\frac{2}{h^{7}}\left[a_{02 x} b_{01} b_{02}+a_{02} b_{02} b_{01 x}-2 b_{01} a_{02}^{2}-\frac{6 a_{02} b_{01} b_{02} h_{x}}{h}\right] \text {. }
\end{aligned}
$$


Inspection of the inhomogeneous part of (6.51) shows that it is a polynomial of $y$. Thus, there is hope to integrate (6.51) analytically. Indeed, after lengthy calculations, the solution of (6.48) subject to the boundary conditions (6.35) turns out to be given by

$$
\begin{aligned}
\Psi_{2}= & \mathscr{R e}\left[\frac{c_{11} y^{11}}{7920 h^{7}}+\frac{c_{12} y^{9}}{3024 h^{5}}+\frac{c_{13} y^{7}}{840 h^{3}}+\frac{c_{14} y^{5}}{120 h}\right]+2 \lambda_{1}\left(\lambda_{1}-\lambda_{2}\right)\left[\frac{m_{1} y^{7}}{42}+\frac{m_{2} y^{5}}{20}+\frac{m_{3} y^{3}}{6}\right] \\
& +2\left(\lambda_{1}-\lambda_{2}\right)\left[\frac{m_{4} y^{10}}{90}+\frac{m_{5} y^{9}}{72}+\frac{m_{6} y^{8}}{56}+\frac{m_{7} y^{7}}{42}+\frac{m_{8} y^{6}}{30}+\frac{m_{9} y^{5}}{20}+\frac{m_{10} y^{3}}{6}\right] \\
& +\frac{m_{11} y^{5}}{20}+\frac{m_{12} y^{3}}{6}+\widetilde{C}_{1} \frac{y^{3}}{6}+\widetilde{C}_{2} y,
\end{aligned}
$$

where

$$
\begin{aligned}
& m_{1}=\frac{1}{5}\left[\frac{d_{11 x}}{h^{12}}-\frac{12 d_{11} h_{x}}{h^{13}}+\frac{d_{13 x}}{h^{12}}-\frac{12 d_{13} h_{x}}{h^{13}}\right]-\frac{2 b_{01} g_{14}}{h^{12}} \\
& +\frac{4 b_{01}^{2}}{h^{8}}\left[\frac{a_{01 x}}{h^{4}}-\frac{4 a_{01} h_{x}}{h^{5}}\right]-\frac{g_{11}}{2 h^{12}}-\frac{J_{11}}{h^{5}}, \\
& m_{2}=\frac{1}{3}\left[\frac{d_{12 x}}{h^{10}}-\frac{10 d_{12} h_{x}}{h^{11}}+\frac{d_{14 x}}{h^{10}}-\frac{10 d_{14} h_{x}}{h^{11}}\right]-\frac{2 b_{01} g_{15}}{h^{8}} \\
& +\frac{4 b_{01}^{2}}{h^{8}}\left[\frac{a_{02 x}}{h^{2}}-\frac{2 a_{02} h_{x}}{h^{3}}\right]-\frac{g_{12}}{2 h^{10}}-\frac{J_{12}}{h^{3}}, \\
& m_{3}=-\left[\frac{2 b_{01} g_{16}}{h^{4}}+\frac{g_{13}}{2 h^{8}}+\frac{J_{13}}{h}\right], \quad m_{4}=-\frac{e_{14}}{28 h^{6}} \text {, } \\
& m_{5}=\frac{14 b_{01} a_{11}}{h^{11}}+\frac{18 a_{01} b_{1}}{h^{11}}+\frac{c_{11}}{2 h^{7}}, \quad m_{6}=-\frac{e_{15}}{15 h^{4}}, \\
& m_{7}=\frac{10 b_{01} a_{12}}{h^{9}}+\frac{6\left(a_{02} b_{1}+2 a_{01} b_{2}\right)}{h^{9}}+\frac{c_{12}}{2 h^{5}}, \quad m_{8}=-\frac{e_{16}}{6 h^{2}}, \\
& m_{9}=\frac{6 b_{01} a_{13}}{h^{7}}+\frac{2\left(2 a_{02} b_{2}+3 a_{01} b_{3}\right)}{h^{7}}+\frac{c_{13}}{2 h^{3}}, \\
& m_{10}=\frac{2 b_{01} a_{14}}{h^{5}}+\frac{2 a_{02} b_{3}}{h^{5}}+\frac{c_{14}}{2 h}, \\
& m_{11}=-2\left[\frac{a_{01 x}}{h^{4}}-\frac{4 a_{01} h_{x}}{h^{5}}\right], \quad m_{12}=-4\left[\frac{a_{02 x}}{h^{2}}-\frac{2 a_{02} h_{x}}{h^{3}}\right] \text {, } \\
& \widetilde{C}_{1}=-\frac{3}{h^{3}}\left[F_{2}+\mathscr{R} h^{4} m_{13}+2 \lambda_{1}\left(\lambda_{1}-\lambda_{2}\right) m_{14} h^{3}\right. \\
& \left.+2\left(\lambda_{1}-\lambda_{2}\right) m_{15} h^{3}+m_{16} h^{5}+m_{17} h^{3}\right],
\end{aligned}
$$




$$
\begin{aligned}
\widetilde{C}_{2}= & \frac{3}{2 h} F_{2}+\mathscr{R e} h^{3} m_{18}+2 \lambda_{1}\left(\lambda_{1}-\lambda_{2}\right) m_{19} h^{2} \\
& +2\left(\lambda_{1}-\lambda_{2}\right) m_{20} h^{2}+m_{21} h^{4}+m_{22} h^{2}, \\
m_{13}= & \frac{9 c_{11}}{7003}+\frac{21 c_{12}}{7938}+\frac{3 c_{13}}{420}+\frac{c_{14}}{30}, \quad m_{14}=\frac{m_{1} h^{4}}{7}+\frac{m_{2} h^{2}}{5}+\frac{m_{3} h}{3}, \\
m_{15}= & \frac{m_{4} h^{7}}{10}+\frac{m_{5} h^{6}}{9}+\frac{m_{6} h^{5}}{8}+\frac{m_{7} h^{4}}{7}+\frac{m_{8} h^{3}}{6}+\frac{m_{9} h^{2}}{5}+\frac{m_{10}}{3}, \\
m_{16}= & \frac{m_{11}}{5}, \quad m_{17}=\frac{m_{12}}{3}, \quad c_{18}= \\
m_{13} & \frac{3 m_{11}}{720}-\frac{c_{12}}{336}-\frac{c_{13}}{120}-\frac{c_{14}}{24}, \\
m_{19}= & \frac{3 m_{14}}{2}-\frac{m_{1} h^{4}}{6}-\frac{m_{2} h^{2}}{4}-\frac{m_{3}}{2}, \\
m_{20}= & \frac{3 m_{15}}{2}-\frac{m_{4} h^{7}}{9}-\frac{m_{5} h^{6}}{8}-\frac{m_{6} h^{5}}{7}-\frac{m_{7} h^{4}}{6}-\frac{m_{8} h^{3}}{5}-\frac{m_{9} h^{2}}{4}-\frac{m_{10}}{2}, \\
m_{21}= & \frac{3 m_{16}}{2}-\frac{m_{11}}{4}, \quad m_{22}=\frac{3 m_{17}}{2}-\frac{m_{12}}{2} .
\end{aligned}
$$

The axial velocity at this order is given by

$$
\begin{aligned}
u_{2}= & \mathscr{R e}\left[\frac{c_{11} y^{10}}{720 h^{7}}+\frac{c_{12} y^{8}}{336 h^{5}}+\frac{c_{13} y^{6}}{120 h^{3}}+\frac{c_{14} y^{4}}{24 h}\right] \\
& +2 \lambda_{1}\left(\lambda_{1}-\lambda_{2}\right)\left[\frac{m_{1} y^{6}}{6}+\frac{m_{2} y^{4}}{4}+\frac{m_{3} y^{2}}{2}\right] \\
& +2\left(\lambda_{1}-\lambda_{2}\right)\left[\frac{m_{4} y^{9}}{9}+\frac{m_{5} y^{8}}{8}+\frac{m_{6} y^{7}}{7}+\frac{m_{7} y^{6}}{6}+\frac{m_{8} y^{5}}{5}+\frac{m_{9} y^{4}}{4}+\frac{m_{10} y^{2}}{2}\right] \\
& +\frac{m_{11} y^{4}}{4}+\frac{m_{12} y^{2}}{2}+\widetilde{C}_{1} \frac{y^{2}}{2}+\widetilde{C}_{2} .
\end{aligned}
$$

It obviously depends on non-Newtonian (elastic) material behavior as it depends on the difference of the Weissenberg numbers.

Such a dependence does now also arise in the pressure rise per wavelength along the central line of the channel $(y=0)$. It can be obtained by integrating the second-order pressure gradient $d p_{2} / d x$ from (6.52):

$$
\triangle P_{\lambda_{2}}=\left.\int_{0}^{2 \pi} \frac{d p_{2}}{d x}\right|_{y=0} d x
$$


After lengthy calculations, the pressure rise per wavelength along the central line of the channel $(y=0)$ at this order is given by

$$
\triangle P_{\lambda_{2}}=\int_{0}^{2 \pi} \tilde{\Phi} d x+6 \lambda_{1}\left(\lambda_{1}-\lambda_{2}\right) \int_{0}^{2 \pi} \widehat{\Phi} d x+6\left(\lambda_{1}-\lambda_{2}\right) \int_{0}^{2 \pi} \Phi^{*} d x
$$

where

$$
\begin{aligned}
& \widetilde{\Phi}=\frac{\mathscr{R} \mathrm{e}^{2}}{560}\left[h_{x x}\left(-\frac{45 F_{0}^{3}}{h^{2}}-\frac{48 F_{0}^{2}}{h}-20 F_{0}-3 h\right)+\left(h_{x}\right)^{2}\left(\frac{90 F_{0}^{3}}{h^{3}}+\frac{48 F_{0}^{2}}{h^{2}}-3\right)\right]-\frac{3 F_{2}}{h^{3}} \\
& +\frac{3}{2} \mathscr{R e} F_{1}\left[\frac{3 F_{0}}{h^{3}}+\frac{1}{2 h^{2}}\right] h_{x}+3 F_{0}\left[\frac{\left(h_{x}\right)^{2}}{h^{3}}-\frac{h_{x x}}{h^{2}}\right] \\
& +\frac{6}{5}\left[\frac{a_{01 x}}{h^{4}}-\frac{4 a_{01} h_{x}}{h^{5}}\right]-\left[\frac{a_{02 x}}{h^{2}}-\frac{2 a_{02} h_{x}}{h^{3}}\right] \\
& -\frac{3 \mathscr{R} \mathrm{e}}{h^{3}}\left[\frac{9}{7003}\left(40 a_{11} b_{01}-24 a_{01} b_{1}\right)+\frac{21}{7938}\left(42 a_{11} b_{02}+18 b_{01} a_{12}-30 a_{02} b_{1}-6 a_{01} b_{2}\right)\right. \\
& \left.+\frac{17}{420}\left(6 b_{02} a_{13}-2 a_{02} b_{3}-6 a_{01}\left(b_{1}+b_{2}+b_{3}\right)-2 b_{01} a_{14}\right)\right], \\
& \widehat{\Phi}=\left(\frac{12 d_{11} h_{x}}{35 h^{9}}-\frac{d_{11 x}}{35 h^{8}}\right)+\left(\frac{2 d_{12} h_{x}}{3 h^{9}}-\frac{d_{12 x}}{15 h^{8}}\right)+\left(\frac{12 d_{13} h_{x}}{35 h^{9}}-\frac{d_{13 x}}{35 h^{8}}\right)+\left(\frac{2 d_{14} h_{x}}{3 h^{9}}-\frac{d_{14 x}}{15 h^{8}}\right) \\
& +\frac{4 b_{01}^{2} a_{01 x}}{7 h^{8}}-\frac{40 b_{01}^{2} a_{01} h_{x}}{7 h^{9}}+\frac{18 a_{01}^{2} b_{01}}{7 h^{8}}+\left(\frac{b_{01}}{14}+\frac{b_{02}}{10}\right) \frac{f_{11 x}}{h^{8}}-4\left(\frac{b_{01}}{7}+\frac{b_{02}}{5}\right) \frac{f_{11} h_{x}}{h^{9}} \\
& -3\left(\frac{a_{01}}{14}+\frac{a_{02}}{10}\right) \frac{f_{11}}{h^{8}}+\left(\frac{b_{01}}{10 h}+\frac{b_{02}}{6}\right) \frac{f_{12 x}}{h^{7}}-\left(\frac{3 b_{01}}{5 h^{5}}+b_{02}\right) \frac{f_{12} h_{x}}{h^{8}}-\left(\frac{a_{01}}{10 h}+\frac{a_{02}}{6}\right) \frac{f_{12}}{h^{7}} \\
& +\frac{6 a_{01} b_{01} b_{01 x}}{7 h^{8}}+\frac{12 a_{01 x} b_{01} b_{02}}{5 h^{8}}-\frac{72 a_{01} b_{01} b_{02} h_{x}}{5 h^{9}}-\frac{4 a_{02 x} b_{01}^{2}}{5 h^{8}}+\frac{4 a_{01} b_{01} a_{02}}{5 h^{8}} \\
& +\frac{2 a_{02} b_{01} b_{01 x}}{5 h^{8}}+\frac{6 a_{01} b_{02} b_{01 x}}{5 h^{8}}+\frac{4 a_{02 x} b_{01} b_{02}}{3 h^{7}}-\frac{16 a_{02} b_{01} b_{02} h_{x}}{3 h^{8}}+\frac{2 b_{01 x} a_{02} b_{02}}{3 h^{7}}, \\
& \Phi^{*}=\frac{h}{280}\left(e_{11 x}-\frac{6 e_{11} h_{x}}{h}\right)+\frac{h}{120}\left(e_{12 x}-\frac{4 e_{12} h_{x}}{h}\right)+\frac{h}{36}\left(e_{13 x}-\frac{2 e_{13} h_{x}}{h}\right) \\
& -\frac{34 a_{11} b_{01}}{9 h^{5}}-\frac{a_{01} b_{1}}{15 h^{5}}-\frac{17 a_{12} b_{01}}{7 h^{5}}+\frac{9 a_{02} b_{1}}{7 h^{5}}-\frac{24 a_{01} b_{2}}{35 h^{5}}-\frac{3 a_{11} b_{02}}{h^{5}}-\frac{6 a_{13} b_{01}}{5 h^{5}} \\
& -\frac{4 a_{02} b_{2}}{5 h^{5}}-\frac{3 a_{01} b_{3}}{5 h^{5}}-\frac{7 a_{14} b_{01}}{15 h^{5}}-\frac{3 a_{13} b_{02}}{5 h^{5}}+\frac{a_{02} b_{3}}{5 h^{5}} \text {. }
\end{aligned}
$$


Summary. We summarize our results of the perturbation series through order 2. Recalling (6.36)-(6.39), (6.46)-(6.50), and (6.52)-(6.59), we have shown that

$$
\begin{aligned}
\Psi= & \left\{-\frac{3}{2}\left(F_{0}+h\right)\left(\frac{L^{3}}{3}-L\right)-y\right\}+\delta\left\{b_{1}\left(\frac{L^{7}}{7}-L\right)+b_{2}\left(\frac{L^{5}}{5}-L\right)+b_{3}\left(\frac{L^{3}}{3}-L\right)\right\} \\
& +\delta^{2} \Psi_{2}, \\
u= & \left\{-\frac{3}{2 h}\left(F_{0}+h\right)\left(L^{2}-1\right)-1\right\}+\frac{\delta}{h}\left[b_{1}\left(L^{6}-1\right)+b_{2}\left(L^{4}-1\right)+b_{3}\left(L^{2}-1\right)\right]+\delta^{2} u_{2}, \\
\frac{\partial p}{\partial x}= & -3\left(\frac{F_{0}}{h^{3}}+\frac{1}{h^{2}}\right)+\delta\left[\frac{30 b_{1} y^{4}}{h^{7}}+\frac{12 b_{2} y^{2}}{h^{5}}+\frac{2 b_{3}}{h^{3}}+\mathscr{R} \frac{d \tilde{p}_{1}}{d x}+2\left(\lambda_{1}-\lambda_{2}\right) \frac{d \hat{p}_{1}}{d x}\right] \\
& +\delta^{2} \frac{\partial p_{2}}{\partial x}, \\
\frac{\partial p}{\partial y}= & -\frac{3 y h_{x}\left(3 F_{0}+2 h\right)}{h^{4}}, \\
\triangle P_{\lambda}= & \left.-3\left[F_{0} I_{3}+I_{2}\right]-3 \delta F_{1} I_{3}+\delta^{2} \triangle P_{\lambda_{2} .} .63\right)
\end{aligned}
$$

\section{Discussion of some results}

The above equations have been programmed to construct explicit results. In this section, some results of the perturbation solutions (6.61) are graphically displayed.

In Figure 7.1, the pressure drops per wavelength along the flow direction (or the pressure rises against the flow direction) $\Delta P_{\lambda}$ are illustrated in terms of the wave amplitude $\Phi$ of the wall disturbance with various values of the Weissenberg numbers $\lambda_{1}$ (Figure 7.1(a)), $\lambda_{2}$ (Figure 7.1(b)), the dimensionless wavenumber $\delta$ (Figure 7.1(c)), and the total flux $F$ (Figure 7.1(d)), respectively. Firstly, it is obvious that for $\Phi=0$ the pressure drop is independent of the Weissenberg numbers $\lambda_{1}$ and $\lambda_{2}$; in such a case, the flow behaves just as a Newtonian fluid. Secondly, with increasing occlusion $\Phi$ the pressure drop increases, that is, an increasing pressure gradient is needed to push the same flux to pass the channel. Thirdly, increasing the Weissenberg number $\lambda_{1}$ causes a decrease of the pressure drop to maintain the same flux (Figure 7.1(a)), whereas with an increase of the Weissenberg number $\lambda_{2}$ the pressure drop $\Delta P_{\lambda}$ increases (Figure 7.1(b)). Fourthly, the larger the wavenumber $\delta$ is, the less pressure drop is required (Figure 7.1(c)). Furthermore, as expected, the larger the flow flux is, the larger the pressure drop needed to press it to pass the channel will be (Figure 7.1(d)).

The transverse distributions of the longitudinal velocity at the most narrow position of the channel, which is at $x=3 \pi / 2$ with a channel half-width of $h=0.7$ for $\Phi=0.3$, are shown in Figure 7.2 for different values of the Weissenberg numbers $\lambda_{1}$ (Figure 7.2(a)), $\lambda_{2}$ (Figure 7.2(b)), dimensionless wavenumber $\delta$ (Figure 7.2(c)), and total flux $F$ (Figure $7.2(\mathrm{~d}))$. Owing to the limit of the no-slip boundary condition in (5.12), the velocity at the wall has the same value $u(y=h)=-1$ in the wave frame for all values of the parameters. The largest difference occurs near the central line $y=0$. With increasing Weissenberg 

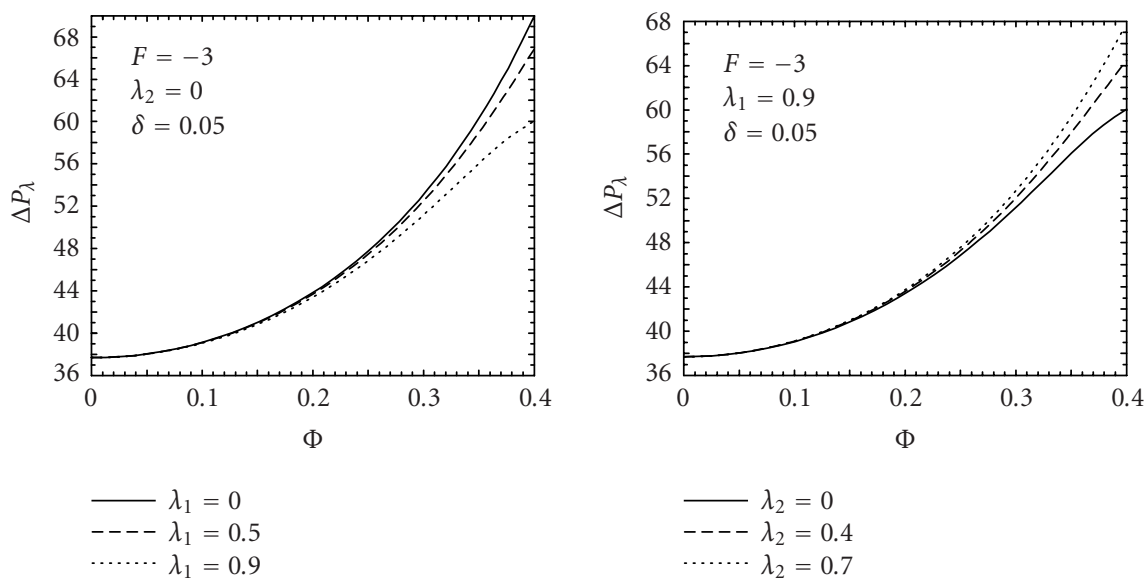

(a)

(b)

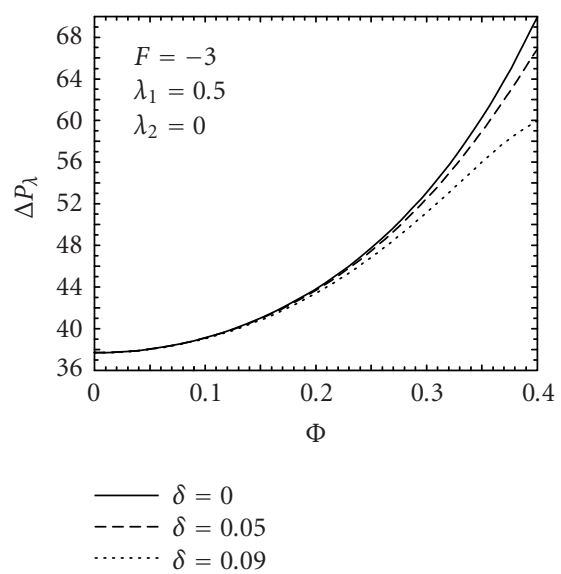

(c)

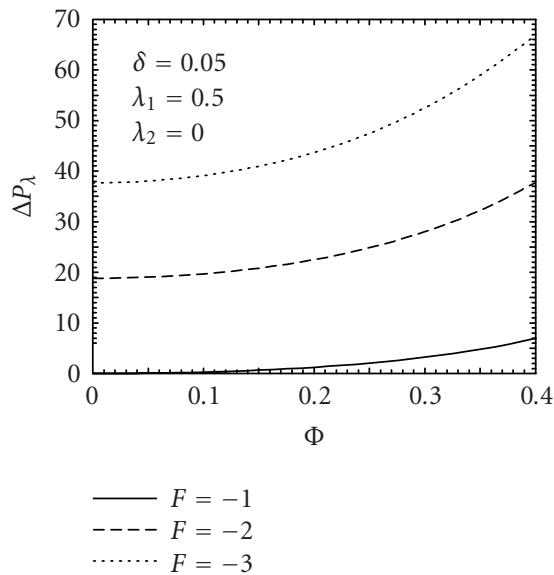

(d)

Figure 7.1. Pressure drop per wavelength at the central axis along the flow direction plotted against the occlusion $\Phi$ for $\mathscr{R} e=1$ with different values of (a) the Weissenberg number $\lambda_{1}$, (b) the Weissenberg number $\lambda_{2}$, (c) the dimensionless wavenumber $\delta$, and (d) the total flux $F$.

number $\lambda_{1}$ (Figure 7.2(a)) and increasing wavenumber $\delta$ (Figure 7.2(c)), the velocity increases, especially near the center of the channel, while an increase of the Weissenberg number $\lambda_{2}$ produces a decrease of the velocity (Figure 7.2(b)). Besides, as a matter of course, a large flux possesses a large velocity, as shown in Figure 7.2(d).

In Figure 7.3, the distributions of the velocity along the central line of the channel $(y=$ 0 ) within a wavelength $x \in(0,2 \pi)$ are displayed for various values of $\lambda_{1}$ and $\delta$. Obviously, 


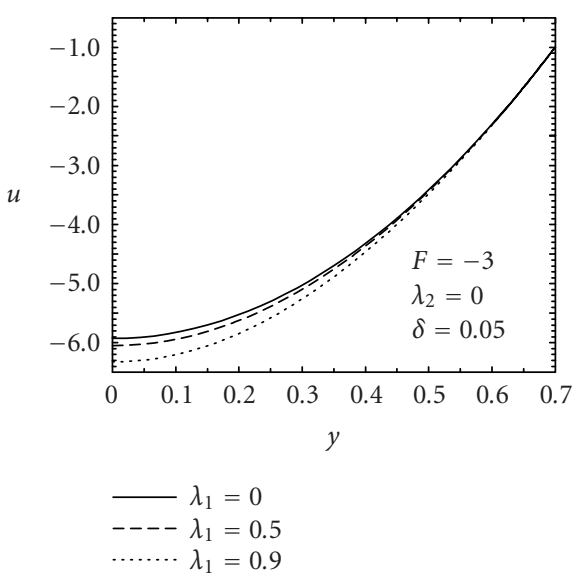

(a)

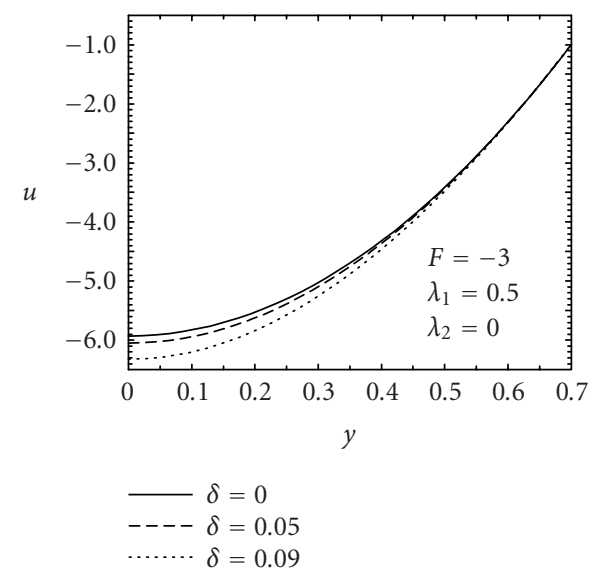

(c)

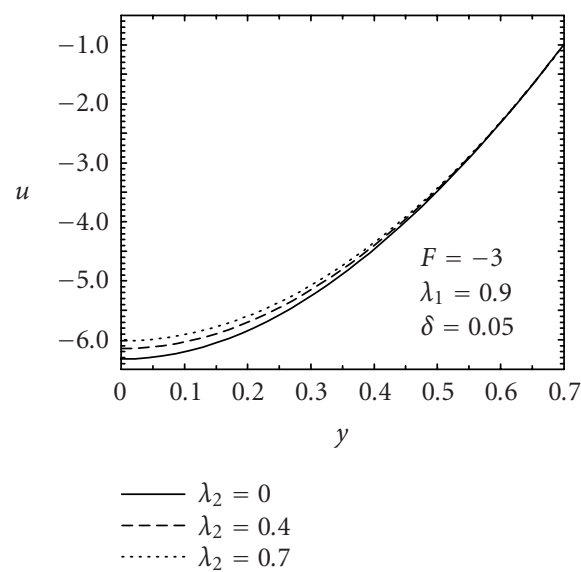

(b)

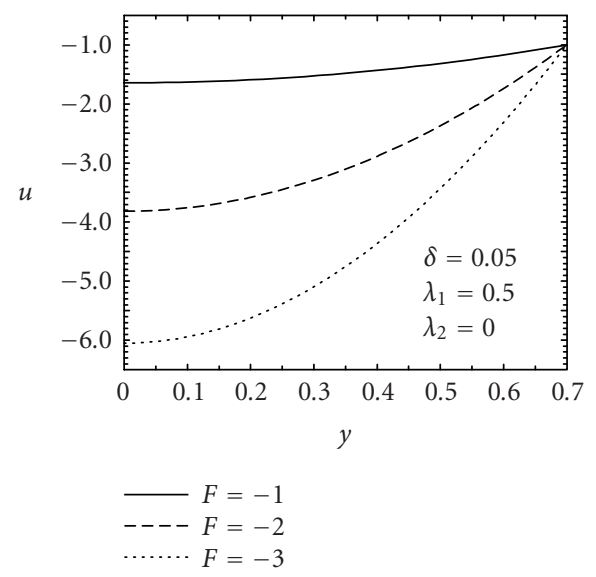

(d)

Figure 7.2. Transverse distribution of the longitudinal (axial) velocity at the most narrow position ( $h=0.7$ when $\Phi=0.3$ ) for $\mathscr{R} e=1$ with different values of (a) the Weissenberg number $\lambda_{1}$, (b) the Weissenberg number $\lambda_{2}$, (c) the dimensionless wavenumber $\delta$, and (d) total flux $F$.

in the narrow part of the channel, $x \in[\pi, 2 \pi]$, especially near the most narrow position $x=3 \pi / 2$, the velocity depends more conspicuously on these parameters than in the wide part of the channel, $x \in[0, \pi]$. As shown in Figure 7.3(a), in the narrow part, the behavior of an Oldroyd-B fluid is obviously different from that of a Newtonian fluid $\left(\lambda_{1}=\lambda_{2}=0\right)$, while in the wide part, their difference may be negligible if the Weissenberg number $\lambda_{1}$ is not extremely large. Similarly, the strong dependence of the velocity on the wavenumber 


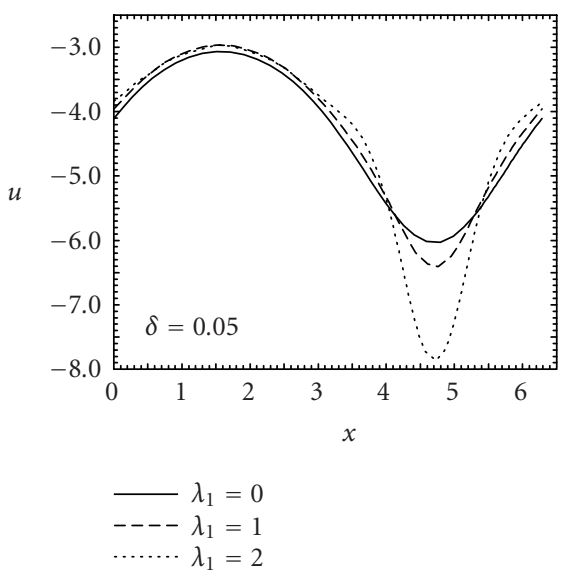

(a)

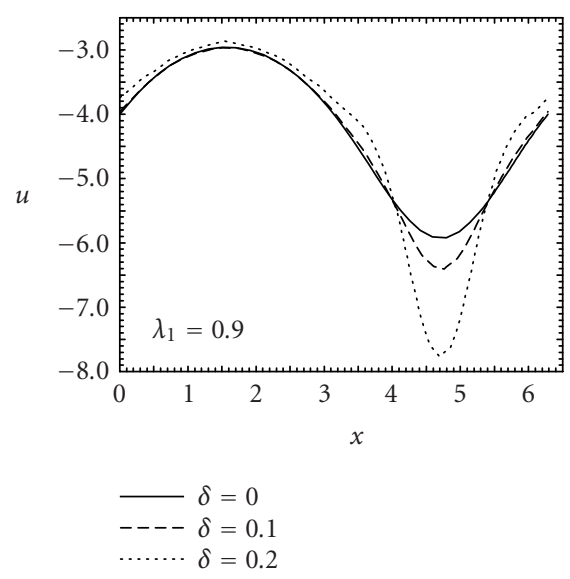

(b)

Figure 7.3. Longitudinal distribution of the axial velocity along the central line of the channel $(y=0)$ with different values of (a) the Weissenberg number $\lambda_{1}$ and (b) the dimensionless wavenumber $\delta$. The other parameters are chosen as follows: $\lambda_{2}=0, F=-3, \Phi=0.3$, and $\mathscr{R} e=1$.

of the wall disturbance occurs also mainly near the narrow part of the channel, as is seen in Figure 7.3(b).

\section{Summary and conclusions}

Peristaltic motion has been studied for two-dimensional geometry in the limit of long wavelength and low frequency. Asymptotic expansions in terms of a dimensionless wavenumber $\delta$ have been constructed, and solutions to $\mathcal{O}\left(\delta^{2}\right)$ have been obtained in closed form.

The limiting solution (i.e., the zeroth-order solution) has been found to be identical to that for an infinite wavelength or Newtonian behavior. At this order, it is found that the Weissenberg numbers only contribute to $S_{0 x x}$. Higher-order solutions have been studied to reveal the effects of non-Newtonian behavior on peristaltic waves with long but finite wavelengths. The results indicate that, for the first-order solution, the Weissenberg numbers do not include any contribution to the stream function, the axial velocity, and pressure rise per wavelength; however, these do give rise to contributions to the longitudinal pressure gradient. The solution for terms of $O\left(\delta^{2}\right)$ depends strongly upon the Weissenberg numbers.

It is hoped that the present analysis may be used with confidence to describe physiological flows in humans with proper geometric modifications.

The following results are found.

(i) The perturbation analysis is valid for large values of wavelength.

(ii) The solution determined here holds for all values of the Reynolds number and the Weissenberg numbers. 
(iii) The terms of orders $\mathscr{O}\left(\delta^{2}\right), \mathcal{O}\left(\delta^{2} \mathscr{R} e^{2}\right), \mathcal{O}\left(\delta^{2} \mathscr{R} e\right), \mathcal{O}\left[\lambda_{1}\left(\lambda_{1}-\lambda_{2}\right) \mathscr{R} e \delta^{2}\right]$, and $\mathscr{O}\left[\left(\lambda_{1}-\right.\right.$ $\left.\left.\lambda_{2}\right) \mathscr{R} e \delta^{2}\right]$ represent, respectively, the curvature, inertia, and the non-Newtonian character of the fluid.

(iv) With increasing occlusion $\Phi$, the pressure drop per wavelength along the flow direction increases, that is, an increase of the pressure gradient is needed to push the same flux to pass the channel.

(v) With a decrease of the Weissenberg number $\lambda_{1}$ or an increase of the Weissenberg number $\lambda_{2}$, the pressure drop per wavelength required to maintain the same flux increases.

(vi) In the narrow part of the channel, the behavior of an Oldroyd-B fluid is much more different from that of a Newtonian fluid than in the wide part of the channel.

(vii) The results for a Maxwell fluid can be obtained as a special case of the presented analysis by taking $\lambda_{2}=0$. To the best of our knowledge, the peristaltic motion of Maxwell fluid has not been discussed so far.

\section{Acknowledgment}

T. Hayat wishes to acknowledge the support of the Alexander von Humboldt Foundation (Bonn, Germany) for this research.

\section{References}

[1] K. Ayukawa, T. Kawa, and M. Kimura, Streamlines and pathlines in peristaltic flows at high Reynolds numbers, Bull. Japan Soc. Mech. Engrs. 24 (1981), 948-955.

[2] K. Ayukawa and S. Takabatake, Numerical analysis of two-dimensional flow, Bull. JSME 25 (1982), 1061-1069.

[3] C. Barton and S. Raynor, Peristaltic flow in tubes, Bull. Math. Biophys. 30 (1968), 663-680.

[4] E. Becker, Simple non-Newtonian flows, Advances in Applied Mechanics (C. S. Yih, ed.), Academic Press, New York, 1980, pp. 177-226.

[5] A. Bedford and D. S. Drumheller, Theories of immiscible and structured mixtures, Int. J. Engng. Sci. 21 (1983), 863-960.

[6] R. B. Bird, R. G. Armstrong, and O. Hassager, Dynamics of Polymeric Liquids. Vol.1. Fluid Mechanics, John Wiley \& Sons, New York, 1987.

[7] J. R. Blake and H. Winet, On the mechanics of muco-ciliary transport, Biorheology 17 (1980), no. $1-2,125-134$.

[8] G. Bohme and R. Friedrich, Peristaltic flow of viscoelastic liquids, J. Fluid Mech. 128 (1983), 109-122.

[9] S. Boyarsky, Surgical physiology of the renal pelvis and ureter, Monogr Surg Sci. 16 (1964), 173213.

[10] T. D. Brown and T.-K. Hung, Computational and experimental investigations of two-dimensional nonlinear peristaltic flows, J. Fluid Mech. 83 (1977), 249-272.

[11] G. Bugliarello and J. Sevilla, Velocity distribution and other characteristics of steady and pulsatile blood flow in fine glass tubes, Biorheology 7 (1970), 85-107.

[12] J. C. Burns and T. Parkes, Peristaltic motion, J. Fluid Mech. 29 (1967), 731-743.

[13] T. S. Chow, Peristaltic transport in a circular cylindrical pipe, J. Appl. Mech. 37 (1970), 901-905.

[14] E. C. Eckstein, Experimental and theoretical pressure studies of peristaltic pumping, S.M. thesis, Massachusetts Institute of Technology, Massachusetts, 1970.

[15] Y. C. Fung and C. S. Yih, Peristaltic transport, J. Appl. Mech. 35 (1968), 669-675. 
[16] H. L. Goldsmith and R. Skalak, Hemodynamics, Ann. Rev. Fluid Mech. 7 (1975), 213-247.

[17] R. D. Greep, Histology Blakiston Division, McGraw-Hill, New York, 1966.

[18] M. Hanin, The flow through a channel due to transversally oscillating walls, Israel J. Tech. 6 (1968), 67-71.

[19] R. H. Haynes, Physical basis of the dependence of blood viscosity on tube radius, Am. J. Physiol. 198 (1960), 1193-1200.

[20] C. D. Hill and A. Bedford, A model for erythrocyte sedimentation, Biorheology 18 (1981), no. 2, 255-266.

[21] T. K. Hung and T. D. Brown, Solid-particle motion in two-dimensional peristaltic flows, J. Fluid Mech. 73 (1976), 77-96.

[22] M. Y. Jaffrin, Inertia and streamline curvature effects on peristalsis pumping, Int. J. Engng. Sci. 11 (1973), 681-699.

[23] M. Y. Jaffrin and A. H. Shapiro, Peristaltic pumping, Ann. Rev. Fluid Dynamics 3 (1971), 13-36.

[24] F. Kill, The Function of the Ureter and Renal Pelvis: Pressure Recordings and Radiographic Studies, W. B. Saunders, Philadelphia, 1957.

[25] T. J. Lardner and W. J. Shack, Cilia transport, Bull. Math. Biophys. 34 (1972), no. 3, 325-335.

[26] T. W. Latham, Fluid motion in a peristaltic pump, Master's thesis, Massachusetts Institute of Technology, Massachusetts, 1966.

[27] H. S. Lew, Y. C. Fung, and C. B. Lowenstein, Peristaltic carrying and mixing of chyme in the small intestine (an analysis of a mathematical model of peristalsis of the small intestine), J. Biomech. 4 (1971), no. 4, 297-315.

[28] C.-H. Li, Peristaltic transport in circular cylindrical tubes, J. Biomech. 3 (1970), no. 5, 513-523.

[29] N. Liron, On peristaltic flow and its efficiency, Bull. Math. Biology 38 (1976), no. 6, 573-596.

[30] _ Fluid transport by cilia between parallel plates, J. Fluid Mech. 86 (1978), no. 4, 705726.

[31] P. S. Lykoudis and R. Roos, The fluid mechanics of the ureter from a lubrication point of view, J. Fluid Mech. 43 (1970), 661-674.

[32] O. H. Mahrenholtz, M. G. Mank, and R. U. Zimmermann, The influence of wave form on peristaltic transport, Biorheology 15 (1978), no. 5-6, 501-510.

[33] M. J. Manton, Long-wavelength peristaltic pumping at low Reynolds number, J. Fluid Mech. 68 (1975), 467-476.

[34] F. E. Marble, Dynamics of dusty gases, Ann. Rev. Fluid Mech. 2 (1970), 397-446.

[35] T. K. Mittra and S. N. Prasad, Interaction of peristaltic motion with Poiseuille flow, Bull. Math. Biol. 36 (1974), 127-141.

[36] S. Oka, A physical theory of erythrocyte sedimentation, Biorheology 22 (1985), 315-321.

[37] J. G. Oldroyd, On the formulation of rheological equations of state, Proc. Roy. Soc. London. Ser. A. 200 (1950), 523-541.

[38] H. Schlichting, Boundary Layer Theory, McGraw-Hill, New York, 1968.

[39] J. H. Semans and O. R. Longworthy, Morphology, motility and fertility of spermatozoa recovered from different areas of ligated rabbit epididymis, J. Reproduct. Fertil. 17 (1938), 125-137.

[40] A. H. Shapiro, Pumping and retrograde diffusion in peristaltic waves, Proc. Workshop Ureteral Reflux Children, Nat. Acad. Sci., Washington, DC, 1967.

[41] A. H. Shapiro, M. Y. Jaffrin, and S. L. Weinberg, Peristaltic pumping with long wavelengths at low Reynolds number, J. Fluid Mech. 37 (1969), 799-825.

[42] J. B. Shukla, R. S. Parihar, B. R. P. Rao, and S. P. Gupta, Effects of peripheral-layer viscosity on peristaltic transport of a bio-fluid, J. Fluid Mech. 97 (1980), no. 2, 225-237.

[43] A. M. Siddiqui, A. Provost, and W. H. Schwarz, Peristaltic pumping of a second order fluid in a planar channel, Rheol. Acta 30 (1991), 249-262.

[44] A. M. Siddiqui and W. H. Schwarz, Peristaltic pumping of a third order fluid in a planar channel, Rheol. Acta 32 (1993), 47-56. 
[45] , Peristaltic flow of a second-order fluid in tubes, J. Non-Newtonian Fluid Mech. 53 (1994), 257-284.

[46] S. L. Soo, Development of dynamics of multiphase flow, Int. J. Sci. Engng. 1 (1984), 1-7.

[47] L. M. Srivastava and V. P. Srivastava, Peristaltic transport of blood: Casson model-II, J. Biomech. 17 (1984), no. 11, 821-829.

[48] _ Peristaltic transport of a particle-fluid suspension, Trans. ASME J. Biomechanical Engng. 111 (1989), no. 2, 157-165.

[49] S. Takabatake and K. Ayukawa, Numerical study of two-dimensional peristaltic flows, J. Fluid Mech. 122 (1982), 439-465.

[50] E. A. Trowbridge, The fluid mechanics of blood, University of Strathclyde Seminars Applied Mathematical Analysis: Mathematics in Medicine and Biomechanics, Shiva Math. Ser., vol. 7, Shiva, Nantwich, 1984, pp. 200-217.

[51] A. J. Vander, J. H. Sherman, and D. Luciano, Human Physiology: The Mechanism of Body Function, Tata McGraw-Hill Publishing, New Delhi, 1975.

[52] S. L. Weinberg, A theoretical and experimental treatment of peristaltic pumping and its relation to ureteral function, Ph.D. thesis, Massachusetts Institute of Technology, Massachusetts, 1970.

[53] S. L. Weinberg, E. C. Eckstein, and A. H. Shapiro, An experimental study of peristaltic pumping, J. Fluid Mech. 49 (1971), 461-479.

[54] H. Winet and J. R. Blake, On the mechanics of mucociliary flows. I. Observations of a channel model, Biorheology 17 (1980), 135-150.

[55] F. Yin and Y. C. Fung, Peristaltic waves in circular cylindrical tubes, J. Appl. Mech. 36 (1969), 579-587, errataum in J. Appl. Mech. 37 (1970), 579-587.

[56] T.-F. Zien and S. Ostrach, A long wave approximation to peristaltic motion, J. Biomech. 3 (1970), no. $1,63-75$.

T. Hayat: Department of Mechanics, Darmstadt University of Technology, Hochschulstraße 1, 64289 Darmstadt, Germany

Current address: Department of Mathematics, Quaid-i-Azam University, Islamabad 45320, Pakistan

E-mail address: t_pensy@hotmail.com

Y. Wang: Department of Mechanics, Darmstadt University of Technology, Hochschulstraße 1, 64289 Darmstadt, Germany

E-mail address: wang@mechanik.tu-darmstadt.de

K. Hutter: Department of Mechanics, Darmstadt University of Technology, Hochschulstraße 1, 64289 Darmstadt, Germany

E-mail address: hutter@mechanik.tu-darmstadt.de

S. Asghar: Department of Mathematics, Quaid-i-Azam University, Islamabad 45320, Pakistan

E-mail address: s_asgharpk@yahoo.com

A. M. Siddiqui: Department of Mathematics, Pennsylvania State University, York Campus, York, PA 17403, USA

E-mail address: ams5@psu.edu 


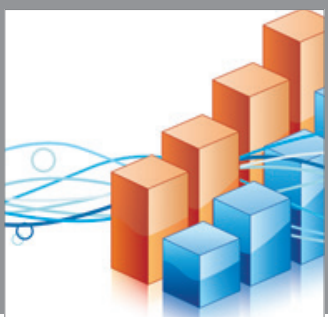

Advances in

Operations Research

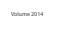

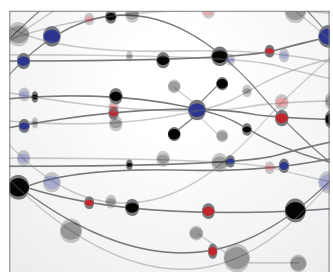

\section{The Scientific} World Journal
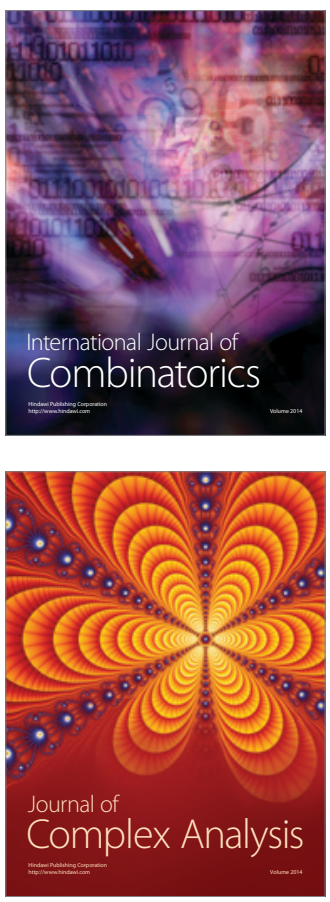

International Journal of

Mathematics and

Mathematical

Sciences
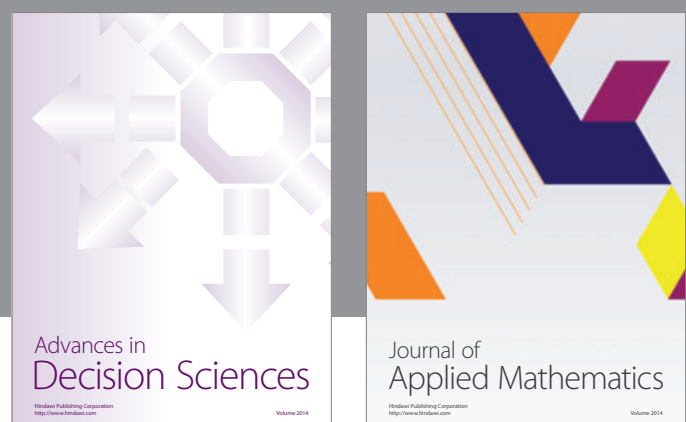

Journal of

Applied Mathematics
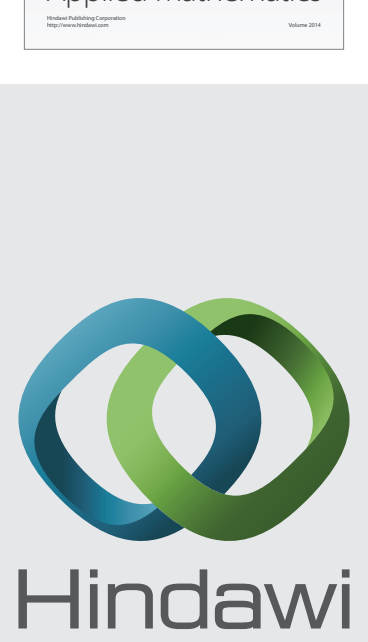

Submit your manuscripts at http://www.hindawi.com
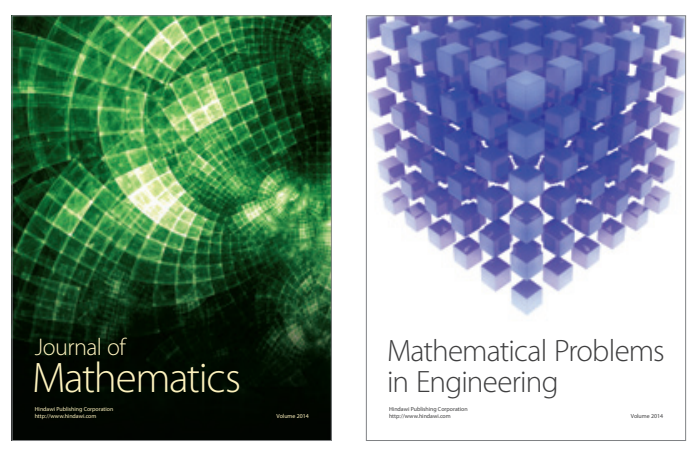

Mathematical Problems in Engineering
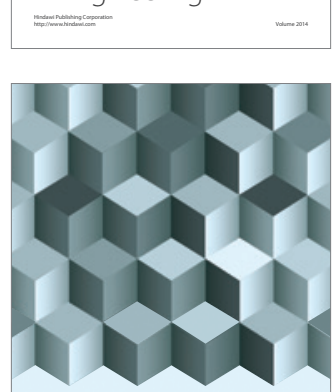

Journal of

Function Spaces
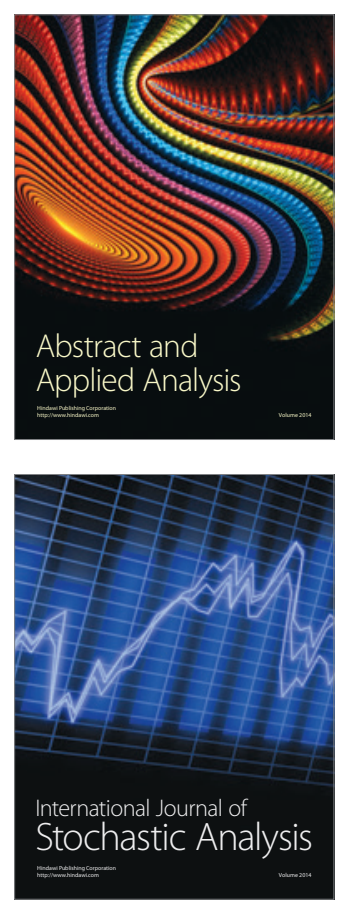

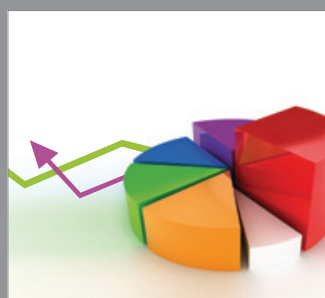

ournal of

Probability and Statistics

Promensencen
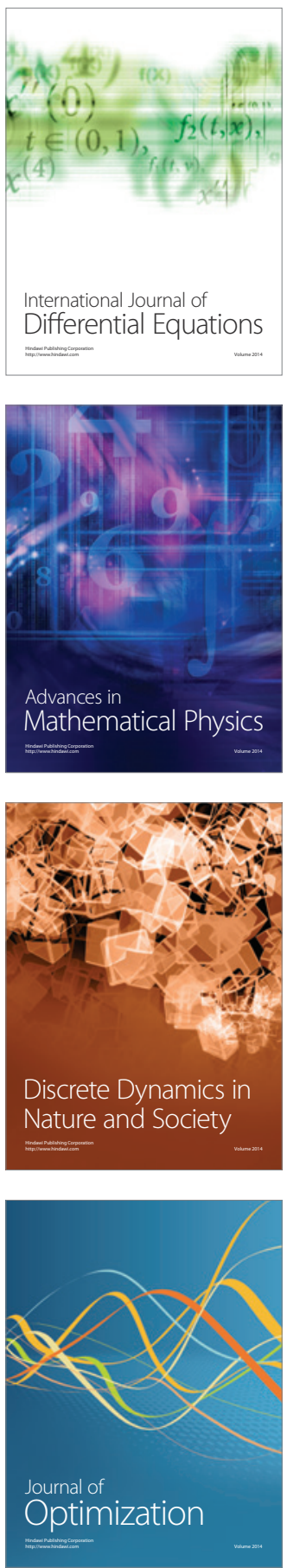